\title{
Quality Control and Calibration of the Dual-Polarization Radar at Kwajalein, RMI
}

David A. Marks (david.a.marks@ nasa.gov); David B. Wolff (david.b.wolff@ nasa.gov)

Lawrence D. Carey (larry.carey@nsstc.uah.edu); Ali Tokay (ali.tokay-1@ nasa.gov)

Article submitted to the Journal of Atmospheric and Oceanic Technology, February 2010

Weather radars, recording information about precipitation around the globe, will soon be significantly upgraded. Most of today's weather radars transmit and receive microwave energy with horizontal orientation only, but upgraded systems have the capability to send and receive both horizontally and vertically oriented waves. These enhanced "dualpolarimetric" (DP) radars peer into precipitation and provide information on the size, shape, phase (liquid / frozen), and concentration of the falling particles (termed hydrometeors). This information is valuable for improved rain rate estimates, and for providing data on the release and absorption of heat in the atmosphere from condensation and evaporation (phase changes). The heating profiles in the atmosphere influence global circulation, and are a vital component in studies of Earth's changing climate. However, to provide the most accurate interpretation of radar data, the radar must be properly calibrated and data must be quality controlled (cleaned) to remove non-precipitation artifacts; both of which are challenging tasks for today's weather radar. The DP capability maximizes performance of these procedures using properties of the observed precipitation.

In a notable paper published in 2005, scientists from the Cooperative Institute for Mesoscale Meteorological Studies (CIMMS) at the University of Oklahoma developed a method to calibrate radars using statistically averaged DP measurements within light rain. An additional publication by one of the same scientists at the National Severe Storms Laboratory (NSSL) in Norman, Oklahoma introduced several techniques to perform quality control of radar data using DP measurements. Following their lead, the Topical Rainfall Measuring Mission (TRMM) Satellite Validation Office at NASA's Goddard Space Flight Center has fine-tuned these methods for specific application to the weather radar at Kwajalein Island in the Republic of the Marshall Islands, approximately 2100 miles southwest of Hawaii and 1400 miles east of Guam in the tropical North Pacific Ocean. This tropical oceanic location is important because the majority of rain, and therefore the majority of atmospheric heating, occurs in the tropics where limited groundbased radar data are available.

The current study supports the conclusions of CIMMS and NSSL that DP radar measurements in light rain provide calibration results very similar to independent, but logistically difficult, techniques. Quantified data reveals that DP-based quality control results in significantly improved data quality and more accurate rainfall measurements. In fact, data contamination from sources such as buildings, towers, ocean waves, and even density changes within the air itself, can be almost completely corrected. The practical application of these DP methods to tropical oceanic radar data provides a calibrated and clean radar dataset crucial to researchers studying our changing planet. 


\title{
Quality Control and Calibration of the Dual-Polarization Radar at Kwajalein, RMI
}

\author{
David A. Marks ${ }^{1,2}$, David B. Wolff ${ }^{1,2}$, Lawrence D. Carey $^{3}$, and Ali Tokay ${ }^{1,4}$ \\ ${ }^{1}$ NASA Goddard Space Flight Center, Laboratory for Atmospheres, Greenbelt, Maryland \\ ${ }^{2}$ Science Systems and Applications, Inc., Lanham, Maryland \\ ${ }^{3}$ Earth System Science Center, University of Alabama in Huntsville, Huntsville, Alabama \\ ${ }^{4}$ Joint Center for Earth Systems Technology, University of Maryland, Baltimore County, \\ Baltimore, Maryland
}

Submitted to

Journal of Atmospheric and Oceanic Technology

February 23, 2010

$\overline{\text { Corresponding author address }}$

David A. Marks

SSAI@NASA/GSFC, Code 613.1

Greenbelt, MD 20771

David.A.Marks@nasa.gov 


\begin{abstract}
The dual-polarization weather radar on Kwajalein Atoll in the Republic of the Marshall Islands (KPOL) is one of the only full-time (24/7) operational S-band dual-polarimetric (DP) radars in the tropics. Through the use of KPOL DP fields and disdrometer data from Kwajalein, quality control (QC) and reflectivity calibration techniques were developed and adapted for use in a near real-time operational environment. Data studies in light rain show that KPOL DP measurements meet or exceed quality thresholds for these applications as determined by consensus of the radar community. While the methodology for development of such applications is well documented, tuning of specific algorithms to the particular regime and observed raindrop size distributions requires a comprehensive testing and adjustment period. Presented are algorithm descriptions and results from five case studies in which QC and absolute reflectivity calibration were performed. Also described is a unique approach to calibrate the differential reflectivity field when vertically pointing scans are not available. Results show the following: 1) DP-based QC provides superior results compared to the legacy Tropical Rainfall Measuring Mission (TRMM) QC algorithm (based on height and reflectivity thresholds), 2) absolute reflectivity calibration can be performed using observations of light rain via a published differential phase-based integration technique; results are within $\pm 1 \mathrm{~dB}$ compared to independent measurements, and 3) a polarimetrically tuned reflectivity-rain rate (Z-R) application extended to six months of data shows very good agreement with independent rain gauge measurements, thereby extolling the benefit of DP-based QC and calibration of the reflectivity field.
\end{abstract}




\section{Introduction and KPOL site description}

Dual-polarimetric (DP) ground-based weather radars are well recognized as vital instruments for applications in hydrology, precipitation microphysics, and hydrometeor identification (Ryzhkov and Zrnic 1998a, Vivekanandan et al. 1999, Straka et al. 2000, Gorgucci et al. 2001, Wang and Carey. 2005, among others); all of which benefit research focusing on ground validation of satellite measurements (Chandrasekar et al. 2008). Kwajalein, Republic of the Marshall Islands (KWAJ) (Figure 1) is an ideal tropical oceanic location for which ground validation, modeling, mesoscale characterization studies, and other activities have focused (Schumacher and Houze 2000, Sobel et al. 2004, Houze et al. 2004, Wolff et al. 2005, Yuter et al. 2005, Blossey et al. 2007, Wang and Wolff, 2009). In support of U.S. Army and Tropical Rainfall Measuring Mission (TRMM) Ground Validation (GV) operations at KWAJ, an S-band DP radar (KPOL) operates on a continual basis, providing unique opportunities for operational algorithm development and adaptation with applications clearly extendable to the Global Precipitation Measurement (GPM) GV program. A goal of the GPM GV program is to improve accuracy of rainfall retrievals by developing and improving physically based radiometer algorithms for application over land and ocean. This approach requires insight into the properties of ice microphysics, parameters of local and regional drop size distributions (DSDs), and delineation between water phases. Through careful observation, DP radars can provide a means to cross-validate parameterized microphysical properties in GPM radiometer retrieval algorithms (Kummerow and Petersen 2006, Chandrasekar et al. 2008). As explained in these references, the utility of any operating radar and rain gauge networks for establishing random and systematic errors in rainfall relative to the GPM core satellite over oceanic domains should be fully explored. Kwajalein is an oceanic site, and the operational DP algorithms for quality 
control (QC), self-consistency calibration, hydrometeor identification, and rainrate estimation provide an opportunity for validation of microphysical properties in ocean-based radiometer retrievals from storm to climate scale. Over land, cloud resolving models (CRMs) are one of several components that will be utilized in the development of ground-based physical retrieval algorithms (Kummerow and Petersen 2006). The operational DP algorithms can be extended to WSR-88D radar sites thereby providing needed input to CRMs with currently parameterized microphysical properties. Deployment of DP diverse WSR-88D radars is scheduled to begin in October 2010 (Istok et al. 2009).

The primary focus of this manuscript is the development and adaptation of operational algorithms for QC and self-consistency calibration with the KPOL radar at Kwajalein for the purpose of exploring DP-based validation capabilities. Section 2 describes the overall quality of the KPOL data as compared to established DP research radars, and details the physically based QC techniques. Section 3 provides a method for calibration of differential reflectivity, and application of self-consistency reflectivity calibration using properties of the rainfall medium. DP calibration results are compared with an independent statistical calibration approach. Finally, the benefit of a DP-based QC'd and calibrated reflectivity field is demonstrated with application of the polarimetrically-tuned Z-R method of Bringi et al. (2004).

\section{KPOL Quality Control}

\section{a. Data quality}

KPOL was upgraded to DP capability in early 1998. Routinely observed parameters and scanning strategies are shown in Tables 1 and 2, respectively. Due to a litany of engineering and mechanical issues with KPOL (Marks et al. 2009), initial DP data were not up to established 
standards of acceptability until 2006. To determine the basic quality of KPOL DP data, empirical comparisons were made with established DP research radars. The National Center for Atmospheric Research (NCAR) S-band polarimetric radar (SPOL) (Lutz et al. 1995) and the Colorado State University - University of Chicago - Illinois State Water Survey (CSU-CHILL) (Brunkow et al. 2000) radar were used as comparison benchmarks to evaluate relative KPOL performance. This was accomplished through analysis of DP measurements in very light rain (20 dBZ $\leq Z_{H} \leq 28 \mathrm{dBZ}$, where $Z_{\mathrm{H}}$ is the horizontal reflectivity component). In this context, drops are essentially spherical with little or no variability in shape, canting angle, or scattering properties within a radar resolution volume (Doviak and Zrnic 1993). Therefore, DP measurements from this medium are used as indicators of data quality. In polarimetric radar applications, the co-polar correlation coefficient $\left(\rho_{\mathrm{HV}}\right)$ is a measure of the correlation between horizontally and vertically polarized weather signals. It is primarily affected by the variability in the ratio of the vertical-to-horizontal size of hydrometeors in the resolution volume, but can also be affected by variability in canting angle and differential phase shift on scattering (Doviak and Zrnic 1993). Canting and scattering properties are of minimal concern in light rain and drizzle due to the mainly spherical shape of the small drops. Theoretical values of $\rho_{\mathrm{HV}}$ larger than 0.99 are indicated by Sachidananda and Zrnic (1985) because of the small shape effects in rain, but theory does not account for possible decreases due to sidelobes and receiver noise. Measurements in rain indicate an average $\rho_{\mathrm{HV}}$ of 0.98 (Doviak and Zrnic 1993) with standard deviation of 0.01 . Therefore, significant deviation $(>0.01)$ of $\rho_{\mathrm{HV}}$ below 0.98 in light rain is a likely indicator of general radar system issues. Other values of polarimetric measurements in light rain (as shown in Doviak and Zrnic 1993 Table 8.1) include the analysis of both specific differential phase $\left(\mathrm{K}_{\mathrm{DP}}\right)$ and differential reflectivity $\left(\mathrm{Z}_{\mathrm{DR}}\right)$, where median $\mathrm{K}_{\mathrm{DP}}$ measurements 
should be approximately $0^{\circ} \mathrm{km}^{-1}$, and deviation of $Z_{\mathrm{DR}}$ from its average value should be approximately $0 \mathrm{~dB}$, i.e.

$$
\sum_{i=1}^{N}\left(Z_{D R}(i)-\overline{Z_{D R}}\right) / N \approx 0 \mathrm{~dB}
$$

where $\mathrm{N}$ is the $\mathrm{Z}_{\mathrm{DR}}$ sample size. DP observations in light rain were compared from the three $\mathrm{S}$ band radars, SPOL, CHILL, and KPOL. Figure 2 shows representative $\rho_{\mathrm{HV}}$, deviation of $\mathrm{Z}_{\mathrm{DR}}$, and $\mathrm{K}_{\mathrm{DP}}$ measurements by relative frequency for specific events from the three radars under typical operating conditions. Base tilt data from representative volume scans were compared. All three panels of Figure 2 indicate that KPOL data quality is at least as good as the established research radars in this specific case. To investigate further, twelve KPOL volume scans from different light rain events from 2006 and 2007 were analyzed with very similar results. To determine if $\mathrm{K}_{\mathrm{DP}}$ data are of sufficient quality for applications of QC and rain rate estimation, phase data were also examined. The standard deviation of measured differential phase, $\sigma\left(\Phi_{\mathrm{DP}}\right)$, was computed at each range gate from a running, centered 25 -gate sample in the radial direction. This corresponds with $0.2 \mathrm{~km}$ gate spacing and the RVP8 $\mathrm{K}_{\mathrm{DP}}$ length scale of $5 \mathrm{~km}$. As discussed in the literature, a reasonable range for $\sigma\left(\Phi_{\mathrm{DP}}\right)$ computations should be approximately $2-3^{\circ}$ or lower for $\mathrm{K}_{\mathrm{DP}}$ applications involving $\mathrm{QC}$, calibration, rain rate estimation and hydrometeor identification (e.g., see Doviak and Zrnic 1993, and Bringi and Chandrasekar 2001 for review). In addition, the absolute average deviation (AAD) of $\mathrm{Z}_{\mathrm{DR}}$ from its mean should provide an upper-end estimate of the random measurement error in $Z_{\mathrm{DR}}$. The $A A D$ of $Z_{\mathrm{DR}}$ is defined by equation 2 , with $\mathrm{N}$ being the number of $\mathrm{Z}_{\mathrm{DR}}$ measurements.

$$
\mathrm{AAD}\left[Z_{\mathrm{DR}}\right]=\sum_{i=1}^{N}\left|\left(Z_{D R}(i)-\overline{Z_{D R}}\right)\right| / N
$$


Table 3 shows statistical relationships between median $\mathrm{Kdp}, \rho_{\mathrm{HV}}, \sigma\left(\Phi_{\mathrm{DP}}\right)$, and $\mathrm{AAD}$ of $Z_{\mathrm{DR}}$ from the CHILL, SPOL, and KPOL radars in light rain. The KPOL statistics represent the range of values from twelve analyzed light rain cases from 2006-2007. These results show that KPOL data quality meets and typically exceeds the radar community's consensus data standards for the implementation of DP radar-based QC, self-consistency calibration, rain rate estimation, and hydrometeor identification algorithms.

\section{b. Radar Data and QC techniques}

KPOL reflectivity and DP data are frequently contaminated by ground and sea clutter, multiple-trip echo, and considerable noise. QC algorithms based on DP measurements have shown notable success in objective identification of these and other non-precipitation features (Ryzhkov and Zrnic 1998b, Zrnic and Ryzhkov 1999, Cifelli et al. 2002). A series of five KPOL case studies with varying rainfall intensities and reflectivity coverage from years 2006 and 2007 were selected to develop an operational QC algorithm for detection and removal of nonprecipitation echo. A monthly study from July 2008 was also performed to check the DP QC algorithm in various other conditions such as isolated to scattered convection and light showers, to periods with only non-precipitation echo present. Since the primary focus was in detection and removal of non-precipitation echo during rain events, the discussion concentrates on results from the five individual case studies. In the following discussion, refer to Table 1 for description of moments and field labels. The multiple steps of the QC algorithm for the first two elevation angles are displayed in flowchart form in Figure 3. For a primary application of quantitative rainfall estimation, the QC steps are applied below the observed radar bright band and sitespecific melting level of approximately 5-6 km (Schumacher and Houze, 2000). In our DP QC algorithm, a new data field with label "CZ" is created for each volume scan and contains the 
final pre-calibrated reflectivity that has been edited for suspected non-precipitation echo. Initially, the $\mathrm{CZ}$ field is simply copied from the raw reflectivity field (ZT) for all elevations scans within a volume. As gates are identified as non-precipitation echo, they are assigned a specific value corresponding to the no-data flag. The first step in the QC process is automated and applied by the RVP8 processor. A signal-to-noise ratio (SNR) test is applied to the $\Phi_{\mathrm{DP}}(\mathrm{PH})$, $\mathrm{K}_{\mathrm{DP}}(\mathrm{KD}), \mathrm{Z}_{\mathrm{DR}}(\mathrm{DR})$, and $\rho_{\mathrm{HV}}(\mathrm{RH})$ fields to identify gates with weak or uncertain signals, and sets the value of these gates to the no-data flag. Multiple-trip echo is usually removed from the $\Phi_{\mathrm{DP}}$ and $\rho_{\mathrm{HV}}$ fields by this technique; however, additional $\mathrm{QC}$ is required for multiple-trip in both $\mathrm{Z}_{\mathrm{H}}$ and $Z_{\mathrm{DR}}$ fields. All gates containing the no-data flag in the $\Phi_{\mathrm{DP}}, \rho_{\mathrm{HV}}, \mathrm{K}_{\mathrm{DP}}$, and $Z_{\mathrm{DR}}$ fields are mapped to the corresponding gates in the QC'd reflectivity field CZ. This step takes advantage of the relatively clean $\Phi_{\mathrm{DP}}$ and $\rho_{\mathrm{HV}}$ fields, and eliminates those gates from $\mathrm{CZ}$ that have been flagged with low SNR by the RVP8 processor. This step also removes gates from CZ for which there are no corresponding DP measurements.

The calculation of $\sigma\left(\Phi_{\mathrm{DP}}\right)$ at each range location, and subsequent threshold comparison, is shown in Ryzhkov and Zrnic (1998b) to be a successful test for detection of anomalous propagation (AP)-induced ground clutter echo. The technique is also effective for any type of ground clutter or region of low SNR, and has been applied to KPOL data for identification of ground clutter associated with human-made structures (i.e. buildings and towers). Before computing the standard deviation, the $\Phi_{\mathrm{DP}}$ field is de-aliased. The total differential phase contains both the radar system phase and a cumulative phase due to scattering from precipitation (Gorgucci et al. 1999; Bringi and Chandrasekar 2001). KPOL system phase has a history of variation from near $0^{\circ}$ to just shy of the maximum unambiguous value of $180^{\circ}$. As a consequence, the $\Phi_{\mathrm{DP}}$ field has shown varying amounts of aliasing depending upon the data 
being analyzed. A gate is considered aliased if the absolute value of the phase difference between consecutive gates exceeds $149^{\circ}$. An additional correlation test is performed to make certain that noise is not being sampled. All aliased gates are corrected by adding $180^{\circ}$ to the existing phase value. Using a running centered 15 -gate sample, $\sigma\left(\Phi_{\mathrm{DP}}\right)$ is then computed at each range gate. If at least 5 of the 15 gates contain valid $\Phi_{\mathrm{DP}}$ measurements, their standard deviation value is assigned to the center of the radial interval; otherwise the $\sigma$ value is set to the no-data flag. The requirement of five or more phase samples for a standard deviation calculation eliminates isolated speckle. When $\sigma\left(\Phi_{\mathrm{DP}}\right)$ calculations are complete for a given sweep, each gate value is checked against an empirically determined threshold. Within precipitation (15 dBZ $\leq$ $\left.\mathrm{Z}_{\mathrm{H}}<55 \mathrm{dBZ}\right)$ the typically observed average $\sigma\left(\Phi_{\mathrm{DP}}\right)$ from KPOL is about $3^{\circ}$. Choosing a multiple of four, the threshold has been set to $12^{\circ}$ and is the same threshold used in Ryzhkov and Zrnic (1998b).

Sea clutter, ground clutter from structures (buildings and towers), and general noise are detected and eliminated by using a combination of $\sigma\left(\Phi_{\mathrm{DP}}\right)$ and $\rho_{\mathrm{HV}}$ thresholding. If a $\sigma\left(\Phi_{\mathrm{DP}}\right)$ gate is greater than the threshold, or has been set to the no-data flag, the corresponding $\mathrm{CZ}$ gate is set to the no-data flag. Similarly with $\rho_{\mathrm{HV}}$, if a correlation gate is less than the threshold of 0.80 , the corresponding $\mathrm{CZ}$ gate is set to the no-data flag. Analysis of $\rho_{\mathrm{HV}}$ within sea clutter reveals values mostly less than 0.40 ; however values in the range from 0.0 to near 0.95 can occur. A similar analysis of $\sigma\left(\Phi_{\mathrm{DP}}\right)$ within sea clutter shows standard deviation values ranging from $3^{\circ}$ to $70^{\circ}$. This wide range of correlation and standard deviation values is expected given the varying nature of returns from ocean waves. With a $\rho_{\mathrm{HV}}$ threshold of 0.80 , and a $\sigma\left(\Phi_{\mathrm{DP}}\right)$ threshold of $12^{\circ}$, almost all sea clutter is detected. Echo clearly identified as ground clutter from reflectivity timeseries analysis displays typical $\rho_{\mathrm{HV}}$ values in the 0.4 to 0.95 range. More than $50 \%$ of these 
ground targets have $\rho_{\mathrm{HV}}$ values exceeding 0.80 , and could easily be incorrectly identified as precipitation echo if the correlation test was considered alone, therefore, the $\sigma\left(\Phi_{\mathrm{DP}}\right)$ test is also needed. Within ground clutter, $\sigma\left(\Phi_{\mathrm{DP}}\right)$ has values ranging from $10^{\circ}$ to near $80^{\circ}$, with a clear majority of values greater than $40^{\circ}$. The combination of the correlation and standard deviation tests identifies almost all ground clutter gates; however a small percentage of problem gates are not flagged by either threshold and survive the QC tests.

An additional series of QC steps to test for realistic values and noise are applied to the $K_{D P}$ and $Z_{D R}$ fields. The $Z_{D R}$ field is calibration corrected prior to this test, and is the topic of the next section. The specific thresholds applied in these tests are appropriate for KPOL, but can easily be tailored to match the nature of site-specific data at other locations. Even in the heaviest rain at Kwajalein $\left(Z_{\mathrm{H}}\right.$ near $\left.52 \mathrm{dBZ}\right), \mathrm{K}_{\mathrm{DP}}$ values do not exceed $2.4^{\circ} \mathrm{km}^{-1}$ as determined from analysis of both radar and disdrometer data. The $\mathrm{K}_{\mathrm{DP}}$ realistic value test considers gates with values $\geq 3^{\circ} \mathrm{km}^{-1}$ or $\leq-2^{\circ} \mathrm{km}^{-1}$ as bad data or noise. The positive end of the $K_{\mathrm{DP}}$ allowed range corresponds to a rain rate of $\sim 136 \mathrm{~mm} \mathrm{hr}^{-1}$ using the $\mathrm{R}\left(\mathrm{K}_{\mathrm{DP}}\right)$ equation of Bringi and Chandrasekar (2001). Analyses of $Z_{D R}$ by reflectivity bin (post calibration) from the lowest two elevation angles $\left(0.4^{\circ}\right.$ and $\left.1.4^{\circ}\right)$ indicate average values approaching $1-1.25 \mathrm{~dB}$ for the heaviest rain, and is a similar $Z_{D R}$ range computed from disdrometer observations (discussed in the following section). For a primary application of quantitative rainfall estimation from the lowest angles, the $\mathrm{Z}_{\mathrm{DR}}$ noise test considers gates with negative values, or those $>2.5 \mathrm{~dB}$ to be suspect. The upper threshold was subjectively determined based on low-elevation KPOL observations under typical operating conditions. These additional tests are needed as spurious $\mathrm{K}_{\mathrm{DP}}$ and $\mathrm{Z}_{\mathrm{DR}}$ gates can be persistent in contaminating the interpolated fields for subsequent rain rate estimation. The percentage of gates flagged as "bad" by each of the QC tests from the first two elevation angles 
and rainfall estimation range of $0-100 \mathrm{~km}$ is shown in Table 4. Within these elevation and range limits, we are well below the melting level and radar observed bright band. The higher percentage of flagged gates for the $Z_{\mathrm{DR}}$ threshold test is due to values less than $0 \mathrm{~dB}$. Gates flagged in total reflectivity (ZT) includes those from the $\rho_{\mathrm{HV}}, \sigma\left(\Phi_{\mathrm{DP}}\right)$, low reflectivity threshold $(<5 \mathrm{dBZ})$, and missing gate tests from the DP fields. The ZT percentages vary significantly from case-to-case and are heavily influenced by flagging those gates less than $5 \mathrm{dBZ}$.

The $\mathrm{CZ}$ field now contains the corrected (pre-calibrated) data. As a final QC step for noise reduction in DP fields, all $\mathrm{CZ}$ gates containing the no-data flag are mapped to the corresponding gates in $\rho_{\mathrm{HV}}, \mathrm{Z}_{\mathrm{DR}}, \mathrm{K}_{\mathrm{DP}}$, and $\Phi_{\mathrm{DP}}$. The first two elevation sweeps are $\mathrm{QC}$ 'd in this manner. Figure 4 shows typical results of the $\mathrm{QC}$ algorithm for the $0.4^{\circ} \mathrm{Z}_{\mathrm{H}}$ field. The top panels $(\mathrm{a}, \mathrm{b})$ of Figure 4 show raw and corrected reflectivity images within $50 \mathrm{~km}$ radius and indicate the effective identification of ground clutter along the atoll perimeter (both embedded and nonembedded) in precipitation echo. It is clear that sea clutter, multiple-trip echo, and general noise have also been identified and removed. The bottom panels (c,d) of Figure 4 show a full $160 \mathrm{~km}$ $0.4^{\circ}$ sweep before and after QC. Pronounced regions of multiple-trip echo (from 220 deg to 250 deg) have been removed in addition to widespread light noise.

A quantitative analysis of ground clutter returns reveals that DP QC results are superior to the legacy TRMM GVS (Ground Validation System non-polarimetric) algorithm. As discussed in Kulie et al. 1999, the GVS QC algorithm identifies non-precipitation echo by use of height and reflectivity threshold parameters, and has a significant weakness in removal of highreflectivity ground clutter, especially when the clutter is near or embedded within precipitation. The strongest precipitation echoes at Kwajalein approach $52 \mathrm{dBZ}$, but ground clutter returns easily exceed this value with measurements ranging from 55 to $70 \mathrm{dBZ}$. Figure 5 shows the 
location of the clutter field at Kwajalein, with 1323 gates (within $50 \mathrm{~km}$ of KPOL) identified as frequent sources of clutter (Silberstein et al. 2008). Reflectivity gates are extracted exclusively from these locations from unedited (raw), and corrected data from both DP and GVS QC algorithms for the five daily case studies. To be reasonably certain that no precipitation echo is selected, only reflectivity values $\geq 55 \mathrm{dBZ}$ are considered to be ground clutter. Table 5 shows the gate count $\geq 55 \mathrm{dBZ}$ from uncorrected, DP corrected, and GVS corrected data. The $19 \mathrm{Dec}$ 2006 case shows that 71 gates from a total of 11907 extracted gates have values $\geq 55 \mathrm{dBZ}$. DP QC has correctly identified and removed all 71 gates (100\% correction), therefore zero clutter gates remain. GVS QC has 53 remaining clutter gates $\geq 55 \mathrm{dBZ}$, roughly corresponding to a $25 \%$ correction. Similar results are shown for all cases. DP QC has virtually no clutter gates remaining, while GVS QC has significant numbers of clutter gates remaining. In these cases, precipitation echo is widespread and covers significant portions of the field, and ground clutter echo is mostly embedded in (or in close proximity to) precipitation echo. The DP QC tests (correlation and standard deviation of phase) detect and remove the embedded clutter, but GVS QC historically fails in this regard. In cases with partial precipitation coverage and nonembedded clutter, it is possible for marginal improvement of GVS QC performance through threshold strengthening, but requires repetitive labor intensive processing. In contrast, the DP QC algorithm is fully automated and provides consistent results without the requirement of parameter adjustments.

\section{KPOL calibration}

a. Differential reflectivity calibration

Accurate $Z_{\mathrm{DR}}$ calibration is essential in the determination of absolute reflectivity calibration $\left(Z_{\mathrm{BIAS}}\right)$ via consistency among the polarimetric variables $\mathrm{Z}_{\mathrm{H}}, \mathrm{Z}_{\mathrm{DR}}$, and $\mathrm{K}_{\mathrm{DP}}$, and is 
also critical for rain rate estimation and hydrometeor identification. Approaches to calibrate the differential reflectivity field can be both engineering oriented and through the use of natural scatterers. The use of vertically pointing (or birdbath) scans in light rain is a favored and reliable approach to determine $Z_{\mathrm{DR}}$ bias (Gorgucci et al 1999, Hubbert et al 2008). The KPOL dataset from years 2006 and 2007 does not contain reliable birdbath scans; therefore an alternative calibration method was needed. Zrnic et al. (2006) describe an approach to partition $Z_{\mathrm{DR}}$ calibration into measurements of constant system biases and time-varying biases with the assumption that the time constant of any slow calibration drift is substantially longer than the duration of a volume scan. Their method is applicable to WSR-88D systems due to the need for calibration results without reliance on vertical profiles in precipitation. This approach is not easily applicable to KPOL without hardware modifications. Hubbert et al. (2003) perform $Z_{\mathrm{DR}}$ calibration by employing a cross-polar power technique from solar measurements. In their approach, solar scans are used to calibrate the receiving path, and the average cross-polar powers for horizontal and vertical orientations are obtained from the backscatter covariance matrix to calibrate the transmission path. However, this technique is not applicable to KPOL due to the unavailability of cross-polar power measurements while operating in simultaneous transmit and receive mode. Using natural properties of scatterers, Ryzhkov et al (2005) analyzed dry aggregates at high elevation angles above the radar-detected melting level. The dry aggregate medium has very low density and therefore has small intrinsic $Z_{D R}$ measurements. This method may be applicable for individual case studies, however, for the operational environment of KPOL the dry aggregate approach was found to yield inconsistent results from scan-to-scan. In practice, it is very difficult to isolate the data above the melting level, avoid contamination from the melting level itself, and yet not be sampling ice medium with preferred orientations. Bechini 
et al. (2008) propose a calibration method based on properties of the rain medium where $Z_{\mathrm{DR}}$ measurements are collected at increasing elevations. The $Z_{\mathrm{DR}}$ profiles are then compared to theoretical profiles to detect and quantify bias. Their method was applied to an operational radar where the scanning strategy prohibited vertical observations.

Instead of developing a theoretical $\mathrm{Z}_{\mathrm{DR}}$ profile as in Bechini et al. (2008), a unique method was applied where the calibration of KPOL $Z_{\mathrm{DR}}$ was accomplished through bias adjustment to a disdrometer-determined $Z_{\mathrm{DR}}$ reference profile. Over $10000 \mathrm{JW}$ disdrometer observations of $\mathrm{Z}_{\mathrm{H}}$ and $\mathrm{Z}_{\mathrm{DR}}$ from 2003 and 2004 were compiled for the reference profile. Assumptions regarding drop size and shape relations used in the disdrometer $\mathrm{Z}_{\mathrm{DR}}$ computation are described in the following section. KPOL $Z_{\mathrm{DR}}$ data from 2006 and 2007 smoothed via a boxcar approach (Bringi and Thurai, 2008) were then calibrated for individual rain events by application of specific offsets as determined by comparison to the disdrometer reference. The $\mathrm{Z}_{\mathrm{DR}} \mathrm{Z}_{\mathrm{H}}$ disdrometer reference profile is shown in the top panel of Figure 6 (bold line with no symbols) together with profiles from five case studies in 2006 and 2007 . The cases were chosen based upon rainfall coverage, and include those with uniform rain shields containing small embedded convective cells. The level of disagreement in $\mathrm{Z}_{\mathrm{DR}}$ distributions within the cases is evident, and their bias relative to the disdrometer reference is shown. Before determining the proper $\mathrm{Z}_{\mathrm{DR}}$ offset, the $\mathrm{Z}_{\mathrm{H}}$ distributions were independently calibrated by the relative calibration adjustment (RCA) method (Silberstein et al. 2008; Marks et al. 2009). The bottom panel of Figure 6 shows the $\mathrm{Z}_{\mathrm{DR}}$ distributions after adjustment to the reference. Emphasis was placed on matching within the $30-40 \mathrm{dBZ}$ range as this represents approximately $85-90 \%$ of the measurements used in self-consistency calibration (section 3b). A trial-and-error adjustment of $\mathrm{Z}_{\mathrm{DR}}$ offsets with comparison to the disdrometer reference resulted in the final offsets to apply in 
each case. While the adjusted $\mathrm{Z}_{\mathrm{DR}}$ profiles are not in perfect agreement, and fluctuations due to sample size limitations are noticeable especially in the upper reflectivity bins, our analysis indicates that the agreement is sufficient to perform a robust calibration of $Z_{H}$ using the selfconsistency technique.

Occasional performance of birdbath scans in light rain commenced in March 2008, and have continued through 2009 . Results consistently show $Z_{\mathrm{DR}}$ running 'hot' by approximately 1.0 dB. Figure 7 displays birdbath scan data from 07 Mar 2008 during a shallow light rain event. The vertical profile of mean $\mathrm{Z}_{\mathrm{DR}}$ (top right panel) shows bias of approximately $+1 \mathrm{~dB}$ at the 3.0 $\mathrm{km}$ level; a height chosen to be above pronounced ground clutter effects and below the melting level. The mean $\rho_{\mathrm{HV}}$ values near 0.99 are indicative of sampling in the rain medium (lower left panel). A $3.0 \mathrm{~km}$ mean $\mathrm{Z}_{\mathrm{DR}}$ distribution by azimuth in the bottom right panel shows the periodic nature of the direction-dependent measurements; an expected structure due to variability in ground clutter response with antenna rotation in the vertical (Gorgucci et al. 1999). $Z_{\mathrm{DR}}$ is averaged from the full azimuth cycle of $360^{\circ}$, over which 1023 samples per azimuth are obtained.

\section{b. Self-consistency calibration: methodology and results \\ i. Methodology}

Absolute radar calibration is a requirement for quantitative rainfall estimation (Ulbrich and Lee 1999). A calibration offset of 2-dB (for example, from 28 to $30 \mathrm{dBZ}$ ) can result in rainfall estimation error of $\sim 30 \%$ as measured by the default WSR-88D reflectivity rain rate relationship (Houze et al. 2004). Some early and relatively simple calibration approaches are described in Atlas (2002). It is well documented that polarimetric properties of the rain medium 
can be used to determine the absolute calibration of a radar system. Techniques to capitalize on these consistency relations range from the comparison of rainfall rates derived from power and phase measurements (Gorgucci et al. 1992), to comparing observed and estimated differential propagation phase (Goddard et al. 1994, Vivekanandan et al. 2003, Ryzhkov et al. 2005, and others). The self-consistency of $Z_{\mathrm{H}}, Z_{\mathrm{DR}}$, and $\mathrm{K}_{\mathrm{DP}}$ measurements was quantified by Scarchilli et al. (1996) and Gorgucci et al. (1999) using a gamma distribution model that described many of the natural variations in the DSD. The majority of these self-consistency calibration techniques have a common necessity of heavy rain rates $\left(>50 \mathrm{~mm} \mathrm{~h}^{-1}\right)$ for significant phase accumulation at S-band over the range profile. Ryzhkov et al. (2005) developed consistency relationships based on existing statistics of DSD measurements and polarimetric radar observations in central Oklahoma, and suggested a methodology for determining absolute reflectivity bias ( $\mathrm{Z}_{\mathrm{BIAS}}$ ) from the self-consistency relation that did not require heavy rainfall at S-band. This methodology compared area-time integrals of measured (processor or user determined) $\mathrm{K}_{\mathrm{DP}}$ and computed (theoretical) $\mathrm{K}_{\mathrm{DP}}$ (as a function of $\mathrm{Z}_{\mathrm{H}}$ and $\mathrm{Z}_{\mathrm{DR}}$ ) and determined $\mathrm{Z}_{\mathrm{BIAS}}$ as the adjustment in $\mathrm{Z}_{\mathrm{H}}$ needed for the integrals to agree.

Integrating $\mathrm{K}_{\mathrm{DP}}$ over a large space-time domain substantially reduces the inherent noisiness of point $\mathrm{K}_{\mathrm{DP}}$ measurements, thereby allowing lighter rains with relatively low $\Phi_{\mathrm{DP}}$ to be acceptable for self-consistency calibration of $Z_{\mathrm{H}}$. Per Ryzhkov et al. (2005), to obtain a selfconsistency $Z_{\mathrm{BIAS}}$ within $1-\mathrm{dB}$, the area-time integral of measured $\mathrm{K}_{\mathrm{DP}}$ should be estimated within $20 \%$ accuracy. To determine the accuracy or standard deviation of $\mathrm{K}_{\mathrm{DP}}$, we use the expression from Balakrishnan and Zrnic (1989) and Carey et al. (2000) (equation A1)

$$
\sigma\left(K_{D P}\right)=\frac{\sqrt{3} \sigma\left(\Phi_{D P}\right)}{N^{1.5} \Delta_{r}}
$$


where $\sigma\left(\Phi_{\mathrm{DP}}\right)$ is the standard deviation of the total differential phase, $\mathrm{N}$ is the number of range gates used in $\mathrm{K}_{\mathrm{DP}}$ calculations, and $\Delta_{\mathrm{r}}$ is the range gate spacing. The length scale for KPOL $\mathrm{K}_{\mathrm{DP}}$ calculations is $5-\mathrm{km}$ and the gate spacing is $0.2 \mathrm{~km}$, therefore, a 25 gate filter is used for each $\mathrm{K}_{\mathrm{DP}}$ calculation. For the five case studies, $\sigma\left(\Phi_{\mathrm{DP}}\right)$ was calculated over 25 gate blocks from all volume scans with careful attention to include data within specified range and azimuth boundaries that contained continuous echo (i.e. phase data from echo edges can be excessively noisy, therefore, these observations are avoided in the standard deviation calculations). The lowest $\sigma\left(\Phi_{\mathrm{DP}}\right)$ observed was $2.40^{\circ}$ from the 19 Dec. case, and the highest was $3.42^{\circ}$ from the 11 Aug. 2007 case. Inserting the lowest and highest $\sigma\left(\Phi_{\mathrm{DP}}\right)$ values in (3), the resulting range of $\sigma\left(\mathrm{K}_{\mathrm{DP}}\right)$ in the data is from $0.17^{\circ} \mathrm{km}^{-1}$ to $0.24{ }^{\circ} \mathrm{km}^{-1}$. As part of the calibration method, $\mathrm{K}_{\mathrm{DP}}$ observations are averaged within each reflectivity bin, and there is an associated reduction in $\sigma\left(\mathrm{K}_{\mathrm{DP}}\right)$ relative to the number of statistically independent samples. The total number of $\mathrm{K}_{\mathrm{DP}}$ samples within each reflectivity bin divided by 25 (block averaging window) provides the number of statistically independent $K_{D P}$ samples. As explained in Ryzhkov et al. (2005), the times reduction in $\sigma\left(\mathrm{K}_{\mathrm{DP}}\right)$ due to averaging is defined as the square root of the number of independent samples. The times reduction is then applied to the $\sigma\left(\mathrm{K}_{\mathrm{DP}}\right)$ high and low values to determine the $\sigma\left(\mathrm{K}_{\mathrm{DP}}\right)$ range in each reflectivity bin. The resulting range of $\sigma\left(\mathrm{K}_{\mathrm{DP}}\right)$ is then compared to the maximum allowed $\sigma\left(\mathrm{K}_{\mathrm{DP}}\right)$ to assess data validity.

This statistical technique was applied to each reflectivity bin from $Z_{\min }(30 \mathrm{dBZ})$ to $Z_{\max }$ (48 dBZ) for all the case studies. The results from the 19 Dec 2006 case are shown in Table 6 and are comparable to the results from all cases. For each reflectivity bin, Table 6 shows the average $K_{D P}$, the number of independent $K_{D P}$ samples, the times reduction in $\sigma\left(K_{D P}\right)$ due to averaging, the maximum allowed $\sigma\left(\mathrm{K}_{\mathrm{DP}}\right)$, and the $\sigma\left(\mathrm{K}_{\mathrm{DP}}\right)$ observed (after reduction from 
averaging). To further clarify using the $30 \mathrm{dBZ}$ bin in Table 6 as an example, the average $\mathrm{K}_{\mathrm{DP}}$ is $0.053^{\circ} \mathrm{km}^{-1}$. There were 43526 total $\mathrm{K}_{\mathrm{DP}}$ samples, so the number of independent samples is 1741 (43526/25), resulting in a $41\left(1741^{0.5}\right)$ times reduction in $\sigma\left(\mathrm{K}_{\mathrm{DP}}\right)$. The range for $\sigma\left(\mathrm{K}_{\mathrm{DP}}\right)$ becomes $0.004{ }^{\circ} \mathrm{km}^{-1}(0.17 / 41)$ to $0.006{ }^{\circ} \mathrm{km}^{-1}(0.24 / 41)$. The maximum allowed $\sigma\left(\mathrm{K}_{\mathrm{DP}}\right)$ is $0.011^{\circ} \mathrm{km}^{-1}$ $\left(20 \%\right.$ of the average $\left.\mathrm{K}_{\mathrm{DP}}\right)$. In this reflectivity bin, and in all bins from all cases, it is shown that the observed range of $\sigma\left(\mathrm{K}_{\mathrm{DP}}\right)$ is below the maximum allowed $\sigma\left(\mathrm{K}_{\mathrm{DP}}\right)$. Due to extensive averaging, the $\mathrm{K}_{\mathrm{DP}}$ data are valid for use in the presented self-consistency calibration method.

The precipitation at Kwajalein is dominated by systems that form in the intertropical convergence zone (ITCZ), and shallow $(<5 \mathrm{~km})$ "warm rain" clouds (Schumacher and Houze 2000, Wolff et al 2005) and is ideal for self-consistency calibration due to this lighter rain regime. To apply the area-integration methodology to KPOL data, DSD measurements from the Kwajalein region were required to derive a consistency equation between $Z_{H}, Z_{D R}$, and $K_{D P}$. Using simulated DSD, Vivekanandan et al. (2003), for example, derived a relationship where $\mathrm{K}_{\mathrm{dp}}$ is expressed as a function of $Z_{\mathrm{H}}$ and $\mathrm{Z}_{\mathrm{DR}}$. In this study, we derived a similar relation using actual disdrometer observations. An impact type Joss-Waldvogel (Joss and Waldvogel 1967) disdrometer sited at Kwajalein from May through December 2003 provided 8779 1-minute resolution DSD measurements (within the 30-48 dBZ interval) to regress the following relation between the variables,

$$
K_{D P}=A Z_{H}^{b} Z_{D R}^{c}
$$

(where $\mathrm{A}=0.17737 \times 10^{-4} ; \mathrm{b}=0.9926$, and $\mathrm{c}=-0.5138$ ) with $\mathrm{Z}_{\mathrm{H}}$ in $\mathrm{mm}^{6} \mathrm{~m}^{-3}, \mathrm{Z}_{\mathrm{DR}}$ in $\mathrm{dB}$, and $\mathrm{K}_{\mathrm{DP}}$ in deg $\mathrm{km}^{-1}$. The polarimetric radar parameters of $Z_{\mathrm{H}}, Z_{\mathrm{DR}}$, and $\mathrm{K}_{\mathrm{DP}}$, were calculated for each minute of DSD observations for an S-band radar $(10.7 \mathrm{~cm})$ and a temperature of $20{ }^{\circ} \mathrm{C}$ as shown in Tokay et al. (2002). For drop shape, the mean axis ratios offered by Andsager et al. (1999) 
were adopted for drops less than $4 \mathrm{~mm}$ in diameter and equilibrium drop shapes (Beard and Chuang 1987) for larger drops. For the fall velocity, we adopted the terminal fall velocity drop diameter relation given by Beard (1976). The coefficient and exponents were derived via a linear least-squares fit regression.

In Ryzhkov et al (2005), their derived consistency equation from DSDs in Oklahoma reduced the impact of variability in the DSD and raindrop shape on the calibration results due to the large-scale integration technique. Following their method, we matched measured $\mathrm{K}_{\mathrm{DP}}$ and computed $\mathrm{K}_{\mathrm{DP}}\left(\mathrm{Z}_{\mathrm{H}}, \mathrm{Z}_{\mathrm{DR}}\right)$ by adjusting $\mathrm{Z}_{\mathrm{H}}$ by an amount (in $\mathrm{dB}$ ) considered as the $\mathrm{Z}_{\mathrm{BIAS}}$. $\mathrm{A}$ practical approach to accomplish this is to divide the data collected from an entire spatial/temporal domain into $1-\mathrm{dB}$ increments of radar reflectivity and compute average values of $K_{\mathrm{DP}}(Z)$ and $Z_{\mathrm{DR}}(Z)$ in each $1-\mathrm{dB}$ interval of $Z$ between $Z_{\min }(30 \mathrm{dBZ})$ and $Z_{\max }(48 \mathrm{dBZ})$. The $\mathrm{Z}_{\mathrm{BIAS}}$ is then determined by matching the integrals

$$
I_{1}=\int_{Z_{\min }}^{Z_{\max }} \overline{K_{D P}(Z)} n(Z) d Z
$$

and

$$
I_{2}=A \int_{Z_{\min }}^{Z_{\max }} Z_{H}^{b}{\overline{Z_{D R}}}^{c} n(Z) d z
$$

with an estimated $\mathrm{Z}_{\mathrm{BIAS}}$ determined from Vivekanandan et al. 2003 by

$$
Z_{\text {bias }}(d B)=10 \log \left(\frac{I_{2}}{I_{1}}\right)
$$

An iterative adjustment approach is required to force agreement of the integrals to within an established bound of $0.1 \mathrm{~dB}$. In all cases analyzed, this has been accomplished with two or less iterations. The same cases analyzed for $\mathrm{QC}$ and $\mathrm{Z}_{\mathrm{DR}}$ calibration were examined for selfconsistency calibration. 
ii Self-consistency calibration: Results and method verification

As a basis for evaluation, the self-consistency results are compared against those from the independent RCA technique (Silberstein et al. 2008). The RCA uses a statistical ensemble of reflectivity values from persistent ground clutter areas from every volume scan to monitor hourly and daily radar sensitivity changes relative to an established baseline. As detailed in Silberstein et al. (2008), the $95^{\text {th }}$ percentile of the clutter area reflectivity distribution at the lowest elevation scan is remarkably stable to within $\pm 0.5 \mathrm{~dB}$, and therefore permits monitoring of radar stability. A practical application of the RCA technique to KPOL data revealed a dramatic improvement in KPOL data stability as evidenced by reflectivity comparison with the TRMM Precipitation Radar (PR). Although the RCA provides a relative calibration, corrected KPOL reflectivity matched the PR to within $\pm 1 \mathrm{~dB}$ on a monthly and yearly basis (Marks et al. 2009; Wang and Wolff, 2009). Table 7 shows self-consistency calibration results as compared to the RCA approach and the absolute value of their difference. From the case studies analyzed, there is agreement between self-consistency and RCA to within $\pm 1 \mathrm{~dB}$. In four cases, the agreement is within 0.5 dB. These results are similar to Ryzhkov et al. (2005) upon comparison of corrected reflectivity with independent measurements. Illingworth and Blackman (2002), and Vivekanandan et al. (2003) have also determined that the accuracy of self-consistency calibration can be as good as 0.5-1 $\mathrm{dB}$ as evaluated through independent comparison. The KPOL results are consistent with previous calibration studies, and provide confidence in the operational method. As explained in Silberstein et al. (2008), there is $\pm 0.5 \mathrm{~dB}$ uncertainty in RCA measurements. Together with the possible $\pm 1 \mathrm{~dB}$ uncertainty shown here, there is a combined calibration uncertainty of $\pm 1.5 \mathrm{~dB}$. 
To avoid spurious values of $\mathrm{K}_{\mathrm{DP}}$ due to adverse effects from non-uniform beam filling (NBF) and have the best possible dataset for $K_{\mathrm{DP}}$ comparison, cross-beam gradients of $\Phi_{\mathrm{DP}}$ should be avoided. NBF may cause perturbations in $\Phi_{\mathrm{DP}}$ (Ryzhkov and Zrnic 1998c; Gosset 2004), subsequently resulting in spuriously large $\mathrm{K}_{\mathrm{DP}}$ (of both signs) in regions of strongly nonuniform precipitation (Ryzhkov 2007). However, for use in an operational environment, data within the range of $20-80 \mathrm{~km}$ from KPOL, and full sweep azimuths $\left(0^{\circ}-360^{\circ}\right)$ were selected with the belief that questionable $\mathrm{K}_{\mathrm{DP}}$ values near echo edges would be statistically insignificant to the final calibration result. As suspected, due to the large amount of integrated data in each case study, the final calibration results using specific range/azimuth thresholding compared to the operational settings were very similar (within $0.25 \mathrm{~dB}$ ).

The consistency relationship given in (4) was derived from disdrometer observations at Kwajalein, and therefore the relationship can serve as a theoretical model for verification of the self-consistency method after bias correction. Figure 8 shows comparisons between average $\mathrm{K}_{\mathrm{DP}}$ measurements (after $Z_{\mathrm{BIAS}}$ correction) and those calculated from self-consistency $\left[\mathrm{K}_{\mathrm{DP}}\left(\mathrm{Z}_{\mathrm{H}}, \mathrm{Z}_{\mathrm{DR}}\right)\right]$. There is generally good agreement between the profiles in most cases (with the 23 Nov. 2007 case being an exception). A noticeable disagreement in profiles is apparent at both lower and upper ends of the reflectivity range. From approximately $30-35 \mathrm{dBZ}$, SIGMET calculated $\mathrm{K}_{\mathrm{DP}}$ is higher than consistency theory $\left(\mathrm{Z}_{\mathrm{H}}, \mathrm{Z}_{\mathrm{DR}}\right.$ model). This could be due to difficulty in extracting the true $\mathrm{K}_{\mathrm{DP}}$ signal from the embedded noise field despite substantial sample size and extensive averaging. The agreement is best within the $35-43 \mathrm{dBZ}$ range, attributed to a stronger $\mathrm{K}_{\mathrm{DP}}$ signal and good sample sizes. In reflectivity bins greater than $43 \mathrm{dBZ}$, the radar measured $\mathrm{K}_{\mathrm{DP}}$ falls lower than the model in all cases. The number of independent $\mathrm{K}_{\mathrm{DP}}$ samples in reflectivity bins from $43-48 \mathrm{dBZ}$ is significantly lower than in the other bins $(<500$ from the 19 Dec case - Table 
6). Probability distribution functions show that in all cases, approximately $95 \%$ of the $\mathrm{K}_{\mathrm{DP}}$ values within the distribution are accounted for within the 30-43 dBZ range. This result is not surprising due to the predominant occurrence of lower reflectivity precipitation at Kwajalein (Schumacher and Houze 2000, Wolff et al. 2005). These calibration results are obviously weighted with the majority of the samples; therefore not much significance can be placed on the results from the higher reflectivity bins. Other explanations for disagreement in profiles at the lower and upper ends of the reflectivity range could be related to possible bias in the disdrometer-derived consistency equation, and effects of processor filtering of differential phase for $\mathrm{K}_{\mathrm{DP}}$ calculation. The relatively poor profile agreement in the 23 Nov. 2007 case is likely related to the nature of the precipitation itself. As compared to the other cases, there is significantly more convection present and the echo shield displays less of a uniform coverage pattern. The most consistent results occur when cases with uniform rain shields are chosen. The calibration results presented in Table 7 and Figure 8 provide further confirmation of the Ryzhkov et al (2005) method, and show that an absolute bias adjustment to $Z_{\mathrm{H}}$ can be determined by matching $\mathrm{K}_{\mathrm{DP}}$ profiles in relatively light rain, provided a local consistency relationship is available.

\section{Application}

A demonstrated application of the benefit of QC'd and calibrated data is presented by estimating rain rate via the polarimetrically-tuned Z-R approach (PTZR) of Bringi et al. (2004), with six months of both corrected and uncorrected radar data. As background reference, the 
Bringi et al. (2004) PTZR approach begins with the assumption of a first-guess Z-R relationship of the form:

$$
Z=a R^{1.5}
$$

The $a$ coefficient in (8) is adjusted on a pixel-by-pixel basis as the DSD and observed DP values evolve in space and time. A normalized gamma DSD is assumed (Testud et al. 2001), with the shape parameter $(\mu)$, median drop diameter $\left(D_{0}\right)$, and concentration $\left(N_{w}\right)$ obtained via S-band polarimetric radar measurements of $\mathrm{Z}_{\mathrm{H}}, \mathrm{Z}_{\mathrm{DR}}$, and $\mathrm{K}_{\mathrm{DP}}$ in different rain rate conditions following the method of Gorgucci et al. (2002). The reflectivity factor $\mathrm{Z}$ is then related to rain rate $\mathrm{R}$ by

$$
Z=\frac{a^{\prime}(\mu)}{\sqrt{N_{w}}} R^{1.5}
$$

where $a^{\prime}(\mu)$ depends on $\mu$ and other constants (derivations in Appendix of Bringi et al. 2004). Finally, they define a new $\hat{a}$ coefficient to replace the coefficient in (8), as:

$$
\hat{a}=\frac{a^{\prime}(\mu)}{\sqrt{N_{w}}}
$$

The new coefficient is calculated at each pixel, and the application of the PTZR to a given pixel is dynamically determined by the magnitude of observed $\mathrm{Z}_{\mathrm{H}}, \mathrm{Z}_{\mathrm{DR}}$, and $\mathrm{K}_{\mathrm{DP}}$. This technique has shown very encouraging results at Kwajalein with QC'd and calibrated data. For example, after application of the PTZR to six months of corrected KPOL data (July-December 2008), the resultant radar accumulations showed very good agreement with fully independent rain gauges, and indicate an absolute bias of only $16.5 \%$ (Figure $9-$ left panel). The results from uncorrected data are also shown in Figure 9 and indicate an absolute bias with rain gauges of 74.5\%. The benefit of calibrated and QC'd data is clearly evident. For this example, the corrected $Z_{\mathrm{H}}$ data have RCA calibration adjustments applied on a daily basis, following the 
method detailed in Marks et al. (2009). What is especially encouraging is the fact that the PTZR approach provides such consistent results without the need of collecting gauge data; a time consuming and costly effort. Gauge data are absolutely necessary for validation of results, but in a regime such as Kwajalein with limited locations for gauge sites, and logistically difficult maintenance and data collection, the PTZR approach can provide high quality near-real-time radar rain estimates when QC'd and calibrated radar data are available.

\section{Summary}

DP radars are a vital tool for GPM validation due to their applications to rainfall microphysical retrievals. The ability to provide consistent and long-term calibrated groundbased DP measurements will prove essential for calibration of the core GPM satellite and for development of physically based passive microwave radiometer algorithms over land. Through the retrieval of DSD parameters relating drop size and shape, rainfall estimation, and hydrometeor identification, the DP radar can provide validation of parameterized microphysical properties.

The development and adaptation of algorithms for QC, calibration, rain rate estimation, and hydrometeor identification to be applied in a continuous, operational environment has been initiated with DP measurements from KPOL in an oceanic environment. Extension of these applications to ground-based WSR-88D radars is expected to begin in late 2010, dependent upon the polarimetric upgrade schedule. Presented are operational algorithms for QC, and absolute reflectivity calibration using polarimetric properties of the rain medium. Application of the QC algorithm has shown to be robust with superior results compared to the standard TRMM GV QC algorithm that employs height and reflectivity thresholds. The ability to detect and remove 
ground and sea clutter embedded in precipitation echo is a distinct advantage of the DP algorithm. Through application of thresholding tests for correlation and standard deviation of differential phase, almost all clutter-type returns are identified and removed. In contrast, the TRMM GV algorithm can remove ground clutter only when not embedded in precipitation, and can be a labor-intensive process. In addition, the RVP8 processor correctly identifies multipletrip echo through an automated SNR power test; a successful result to which the QC algorithm takes full advantage.

A technique to determine $Z_{\mathrm{DR}}$ calibration through analysis of combined $Z_{\mathrm{DR}} \mathrm{Z}_{\mathrm{H}}$ profiles was developed and applied to KPOL data. This application relies on the independent RCA for reflectivity calibration, and adjusts $Z_{D R}$ profiles to match a disdrometer distribution when birdbath scans are not available. By this technique, uncertainty has been mitigated in $\mathrm{Z}_{\mathrm{DR}}$ data from significant rainfall events in 2006 and 2007 and has allowed application of self-consistency reflectivity calibration.

A published phase-based self-consistency approach to determine absolute reflectivity calibration using properties of the rain medium in light rain has been tested with KPOL data from five case studies and found to provide good results (within $\pm 1.0 \mathrm{~dB}$ ) as compared to the independent RCA method. The approach follows the work of Ryzhkov et al. 2005 where $\mathrm{K}_{\mathrm{DP}}$ data from light rain events are integrated and compared against a model consistency equation derived from disdrometer data at Kwajalein. In lower reflectivity bins (30-35 dBZ), the observed $\mathrm{K}_{\mathrm{DP}}$ measurements indicate a high bias relative to the disdrometer-based model, possibly due to difficulty in extracting the true $\mathrm{K}_{\mathrm{DP}}$ signal. In mid-reflectivity bins (36-43 dBZ), there is good agreement between observed and model $\mathrm{K}_{\mathrm{DP}}$ profiles (with one exception). The results indicate 
that the method can be successfully applied to lighter precipitation regimes. The most consistent results were found in cases with uniform rain shields.

The benefit of QC'd and calibrated radar data was demonstrated via rainfall estimation using the polarimetrically-tuned Z-R approach of Bringi et al. (2004). With corrected radar data, rainfall estimates showed very good agreement with independent rain gauges. The absolute bias between radar and gauge accumulations was reduced from $74 \%$ to near $16 \%$ when QC'd and calibrated radar data were used. This result is impressive considering that the Bringi approach does not require gauge data for calibration and is therefore able to provide high-quality radar rain products in near-real-time. Ongoing work will include quantitative rainfall estimation, QC aloft (melting zone and above) and full volume qualitative hydrometeor identification.

\section{Acknowledgements}

This research is supported by NASA Grant NNG07EJ50C. The authors thank Dr. Ramesh Kakar (NASA Headquarters), Dr. Scott Braun (TRMM Project Scientist), and Dr. Mathew Schwaller (NASA/GSFC) for their support of this effort. Appreciation is extended to David Silberstein for numerous helpful discussions, and to the TRMM Satellite Validation Office staff, including Jason Pippitt, David Makofski, Bart Kelley, and Jianxin Wang. 


\section{References}

Andsager, K, K. V. Beard, and N. F. Laird, 1999: Laboratory measurements of axis ratios for large raindrops. J. Atmos. Sci., 56, 2673-2683.

Atlas, D., 2002: Radar calibration: some simple approaches. Bull. Amer. Meteor. Soc., 83, 13131316.

Beard, K. V., 1976: Terminal velocity and shape of cloud and precipitation drops aloft. $J$. Atmos. Sci., 33, 851-864.

Beard, K. V., and C. Chuang, 1987: A new model for the equilibrium shape of drops. J. Atmos. Sci., 44, 1509-1524.

Bechini, R., L. Baldini, R. Cremonini, and E. Gorgucci, 2008: Differential reflectivity calibration for operational radars. J. Atmos. Oceanic Technol., 25, 1542-1555.

Balakrishnan, N., and D. S. Zrnic, 1989: Correction of propagation effects at attenuating wavelengths in polarimetric radars. Preprints, 24th Conf. on Radar Meteorology, Tallahassee, FL, Amer. Meteor. Soc., 287-291.

Blossey, P. N., C. S. Bretherton, J. Cetrone, and M. Kharoutdinov, 2007: Cloud-Resolving Model Simulations of KWAJEX: Model Sensitivities and Comparisons with Satellite and Radar Observations. J. Atmos. Sci., 64, 1488-1508.

Bringi, V. N., and V. Chandrasekar, Polarimetric Doppler Weather Radar-Principles and Applications, 636pp., Cambridge Univ. Press, New York, 2001.

Bringi, V.N., T. Tang, and V. Chandrasekar, 2004: Evaluation of a New Polarimetrically Based Z-R Relation. J. Atmos. Oceanic Technol., 21, 612-623.

Bringi, V. N., and M. Thurai, 2008: Internal report for BMRC-BOM on the preliminary assessment and analysis of CP2 S and X band data, 6 pp. (available from author) 
Brunkow D., V. N. Bringi, P. C. Kennedy, S. A. Rutledge, V. Chandrasekar, E. A. Mueller, and R. K. Bowie, 2000: A description of the CSU-CHILL National Radar Facility. J. Atmos. Oceanic Technol., 17, 1596-1608

Carey, L.D., S.A. Rutledge, D.A. Ahijevych, and T.D. Keenan, 2000: Correcting Propagation Effects in C-Band Polarimetric Radar Observations of Tropical Convection Using Differential Propagation Phase. J. Appl. Meteor., 39, 1405-1433.

Chandrasekar, V., A. Hou, E. Smith, V. N. Bringi, S. A. Rutledge, E. Gorgucci, W. A. Petersen, and G. S. Jackson, 2008: Potential role of dual-polarization radar in the validation of satellite precipitation measurements. Bull Amer. Meteor. Soc., 89, 1127-1145.

Cifelli, R., W. A. Petersen, L. D. Carey, S. A. Rutledge, and M. A. F. da Silva Dias, 2002: Radar observations of the kinematic, microphysical, and precipitation characteristics of two MCSs in TRMM LBA. J. Geophys. Res., 107(D20), 8077, doi:10.1029/2000JD000264.

Doviak, R. J., and D. S. Zrnic, 1993: Doppler radar and weather observations, $2^{\text {nd }}$ ed., 562pp., Academic Press, Inc., San Diego, CA.

Goddard, J., J. Tan, and M. Thurai, 1994: Technique for calibration of meteorological radars using differential phase. Electron. Lett., 30, 166-167.

Gorgucci, E., G. Scarchilli, and V. Chandrasekar, 1992: Calibration of radars using polarimetric techniques. IEEE Trans. Geosci. Remote Sens., 30, 853-858.

Gorgucci, E., G. Scarchilli, and V. Chandrasekar, 1999: A procedure to calibrate multiparameter weather radar using properties of the rain medium. IEEE Trans. Geosci. Remote Sens., 34, 269-276. 
Gorgucci, E., G. Scarchilli, V. Chandrasekar, and V. N. Bringi, 2001: Rainfall estimation from polarimetric radar measurements: composite algorithms immune to variability in raindrop shape-size relation. J. Atmos. Oceanic Technol., 18, 1773-1786.

Gorgucci, E., V. Chandrasekar, V.N. Bringi, and G. Scarchilli, 2002: Estimation of Raindrop Size Distribution Parameters from Polarimetric Radar Measurements. J. Atmos. Sci., 59, $2373-2384$.

Gosset, M., 2004: Effect of Nonuniform Beam Filling on the Propagation of Radar Signals at XBand Frequencies. Part II: Examination of Differential Phase Shift. J. Atmos. Oceanic Technol., 21, 358-367.

Houze, R. A. Jr., S. Brodzik, C. Schumacher, and S. Yuter, 2004: Uncertainties in oceanic radar rain maps at Kwajalein and implications for satellite validation. J. Appl. Meteor., 43, 11141132.

Hubbert, J. C., F. Pratte, M. Dixon, and R. Rilling, 2008: The uncertainty of Zdr calibration. Preprints, European Conference on Radar Meteorology (ERAD 2008). [Available online at http://erad2008.fmi.fi/proceedings/extended/erad2008-0285-extended.pdf.]

Hubbert, J. C., V. N. Bringi, and D. Brunkow, 2003: Studies of the polarimetric covariance matrix. Part I: Calibration methodology. J. Atmos. Oceanic Technol., 20, 696-706.

Illingworth, A., and T. Blackman, 2002: The need to represent raindrop size spectra as normalized Gamma distributions for the interpretation of polarization radar observations. $J$. Appl. Meteor., 41, 286-297. 
Istok, M. J., M. Fresch, Z. Jing, S. Smith, R. Murnan, A. Ryzhkov, J. Krause, M. Jain, P. Schlatter, J. Ferree, B. Klein, D. Stein, G. Cate, and R. Saffle, 2009: WSR-88D dual polarization initial operational capabilities. $25^{\text {th }}$ Conference on Interactive Information and Processing Systems for Meteorology, Oceanography, and Hydrology, AMS 2009.

Joss, J. and A. Waldvogel, 1967: A spectrograph for the automatic analysis raindrops,. Pure Appl. Geophys., 68, 240-246

Kulie, M. S., M. Robinson, D. A. Marks, B. S. Ferrier, D. Rosenfeld, and D. B. Wolff, 1999: Operational processing of ground validation data for the Tropical Rainfall Measuring Mission. Preprints, $29^{\text {th }}$ Int. Conf. on Radar Meteorology, Montreal, QC, Canada, Amer. Meteor. Soc., 736-739.

Kummerow, C., and W. A. Petersen, 2006: GPM ground validation: strategy and efforts. Available from http://gpm.gsfc.nasa.gov/ground_advisory.html.

Lutz, J., P. Johnson, B. Lewis, E. Loew, M. Randall, and J. VanAndel, 1995: NCAR's S-Pol: Portable polarimetric S-band radar, paper presented at 9th Conference on Meteorological Observations and Instrumentation, Am. Meteorol. Soc., Charlotte, N. C., March 27-31, 1995.

Marks, D. A., D. B. Wolff, D. S. Silberstein, A. Tokay, J. L. Pippitt, and J. Wang, 2009: Availability of high-quality TRMM ground validation data from Kwajalein, RMI: A practical application of the relative calibration adjustment technique. J. Atmos. Oceanic Technol., 26, 413-429.

Ryzhkov, A. V., and D. S. Zrnic, 1998a: Discrimination between rain and snow with a polarimetric radar. J. Appl. Meteor., 37, 1228-1240. 
Ryzhkov, A. V., and D. S. Zrnic, 1998b: Polarimetric rainfall estimation in the presence of anomalous propagation. J. Atmos. Oceanic Technol., 15, 1320-1330.

Ryzhkov, A. V., and D. S. Zrnic, 1998c: Beamwidth Effects on the Differential Phase Measurements of Rain. J. Atmos. Oceanic Technol., 15, 624-634.

Ryzhkov, A. V., S. E. Giangrande, V. M. Melnikov, and T. J. Schuur, 2005: Calibration issues of dual-polarization radar measurements. J. Atmos. Oceanic Technol., 22, 1138-1154.

Ryzhkov, A.V., 2007: The Impact of Beam Broadening on the Quality of Radar Polarimetric Data. J. Atmos. Oceanic Technol., 24, 729-744.

Sachidananda, M., and D. S. Zrnic, 1985: ZDR measurement considerations for a fast scan capability radar. Radio Sci. 20, 907-922.

Scarchilli, G., E. Gorgucci, V. Chandrasekar, and A. Dobaie, 1996: Self-consistency of polarization diversity measurement of rainfall. IEEE Trans. Geosci. Remote Sens., 34, 22-26.

Schumacher, C., and R. A. Houze, Jr., 2000: Comparison of radar data from the TRMM satellite and Kwajalein oceanic validation site. J. Appl. Meteor., 39, 2151-2164.

Silberstein, D. S., D. B. Wolff, D. A. Marks, D. Atlas, and J. L. Pippitt, 2008: Ground clutter as a monitor of radar stability at Kwajalein, RMI. J. Atmos Oceanic Technol., 25, 2037-2045.

Sobel, A. H., S.E. Yuter, C.S. Bretherton, and G.N. Kiladis, 2004: Large-Scale Meteorology and Deep Convection during TRMM KWAJEX. Mon. Wea. Rev., 132, 422-444.

Straka, J. M., D. S. Zrnic, and A. V. Ryzhkov, 2000: Bulk hydrometeor classification and quantification using polarimetric radar data: synthesis of relations. J. Appl. Meteor., 39, $1341-1372$. 
Testud, J., S. Oury, R.A. Black, P. Amayenc, and X. Dou, 2001: The concept of "normalized" distribution to describe raindrop spectra: A tool for cloud physics and cloud remote sensing. J. Appl. Meteor., 40, 1118-1140.

Tokay, A., A. Kruger, W. Krajewski, P. A. Kucera, and A. J. Pereira Filho, 2002: Measurements of drop size distribution in the southwestern Amazon basin. J. Geophys. Res., 107, D20, 8052, doi: 10.01029 .

Ulbrich, C. W., and L. G. Lee, 1999: Rainfall measurement error by WSR-88D radars due to variations in Z-R law parameters and the radar constant. J. Atmos. Oceanic Technol., 16, $1017-1024$.

Vivekanandan, J., D. S. Zrnic, S. M. Ellis, R. Oye, A. V. Ryzhkov, and J. Straka, 1999: Cloud microphysics retrieval using S-band dual-polarization radar measurements. Bull. Amer. Meteor. Soc., 80, 381-388.

Vivekanandan, J., G. Zhang, S. Ellis, D. Rajopadhyaya, and S. Avery, 2003: Radar reflectivity calibration using differential propagation phase measurement. Radio Sci., 38, 8049, doi:10.1029/2002RS002676.

Wang, J., and D.B. Wolff, 2009: Comparisons of reflectivities from the TRMM Precipitation Radar and ground-based radars. J. Atmos. Oceanic Technol., 26, 857-875.

Wang, J. J., and L. D. Carey, 2005: The development and structure of an oceanic squall-line system during the South China Sea Monsoon Experiment. Mon. Weather. Rev., 133, 15441561.

Wolff, D. B., D. A. Marks, E. Amitai, D. S. Silberstein, B. L. Fisher, A. Tokay, J. Wang, and J. L. Pippitt, 2005: Ground Validation for the Tropical Rainfall Measuring Mission (TRMM). J. Atmos. Oceanic Technol., 22, 365-379. 
Yuter, S. E., R. A. Houze, Jr., E. A. Smith, T. Wilheit, and E. Zipser, 2005: Physical characterization of tropical oceanic convection observed in KWAJEX. J. Appl. Meteor., 44, $385-415$.

Zrnic, D. S., and A. V. Ryzhkov, 1999: Polarimetry for weather surveillance radars. Bull. Amer. Meteor. Soc., 80, 389-406.

Zrnic, D. S., V. M. Melnikov, and J. K. Carter, 2006: Calibrating differential reflectivity on the WSR-88D. J. Atmos. Oceanic Technol., 23, 944-951. 


\section{List of Figure Captions}

Fig 1. The location of Kwajalein Atoll in the Republic of the Marshall Islands (from Wolff et al. 2005) The KPOL S-band radar is located on Kwajalein Atoll at the center of the image $\left(8.7^{\circ} \mathrm{N}\right.$, $\left.167.7^{\circ} \mathrm{E}\right)$. Rain gauge locations from the KWA network are shown as black squares.

Fig. 2. Comparison of co-polar $\rho_{\mathrm{HV}}$ (top panel), $\mathrm{Z}_{\mathrm{DR}}$ deviation $\left(\mathrm{Z}_{\mathrm{DR}}-\right.$ average $\left.\mathrm{Z}_{\mathrm{DR}}\right)$ (middle), and $\mathrm{K}_{\mathrm{DP}}$ (bottom) polarimetric parameters in light rain from CHILL, SPOL, and KPOL radars. CHILL observations are from 16 July 20042142 UTC, SPOL observations are from 26 Jan. 1999 2139UTC, and KPOL observations are from 11 Aug. 20070352 UTC.

Fig. 3. KPOL quality control algorithm flowchart for the first two elevation angles. All data are below the melting level.

Fig. 4. QC examples showing persistent ground clutter (a) in raw KPOL data along atoll edges (50 km range ring), and removal of embedded and non-embedded ground clutter by the QC

algorithm (b). A raw 0.4 degree elevation sweep (c) shows significant multiple-trip echo (southwest of KPOL, 20-160 km range), sea clutter, and typical noise. The QC'd field (d) shows dramatic improvement. Range rings are at $50 \mathrm{~km}$ intervals.

Fig. 5. Map of the clutter field at Kwajalein. Range rings are drawn at 10-km intervals from the radar site. Figure from Silberstein et al. 2008. 
Fig. 6 Distribution of average $Z_{D R}$ measurements by reflectivity bin. The unadjusted $Z_{D R}$ curves (top panel) show the bias in five case studies relative to the JWD $Z_{\mathrm{DR}}$ reference (bold, no-symbol line). The bottom panel shows $Z_{\mathrm{DR}}$ distributions bias-adjusted to the JWD reference. $Z_{\mathrm{H}}$ distributions have been calibration corrected via the independent RCA method.

Fig. 7. Measurements of reflectivity with height (top left), mean $Z_{D R}$ with height (top right) correlation with height (bottom left), and the mean $Z_{D R}$ profile with azimuth (bottom right) for a shallow light rain event at Kwajalein from 07 Mar 2008. The correlation profile indicates sampling in the rain medium at the $3.0 \mathrm{~km}$ height. The azimuth profile shows the influence of directional-dependent response. The mean $\mathrm{Z}_{\mathrm{DR}}$ bias of $+1.12 \mathrm{~dB}$ (heavy dashed lined) was determined over the full azimuth cycle. The dash-dot lines represent one standard deviation.

Fig. 8. Comparison of radar processor determined $\mathrm{K}_{\mathrm{DP}}$ measurements and computed $\mathrm{Kdp}$ measurements from self-consistency theory. The self-consistency relationship was developed from disdrometer measurements at Kwajalein. Results are shown from each of the five case studies. 
Figure 9: Scatterplots of independent gauge and radar monthly accumulations for the period July-December 2008 at Kwajalein using the polarimetrically-tuned Z-R approach of Bringi et al. (2004). Results show the benefit of quality controlled and calibrated radar data (left panel) vs. uncorrected data (right panel). The absolute bias using corrected and uncorrected radar data are $16.5 \%$ and $74.5 \%$, respectively, with bias calculated as $\frac{\overline{\mid \text { Radar-Gauge }}}{\overline{\text { Gauge }}}$. Regression lines (solid) are shown for each dataset along with the 1-to-1 line. 


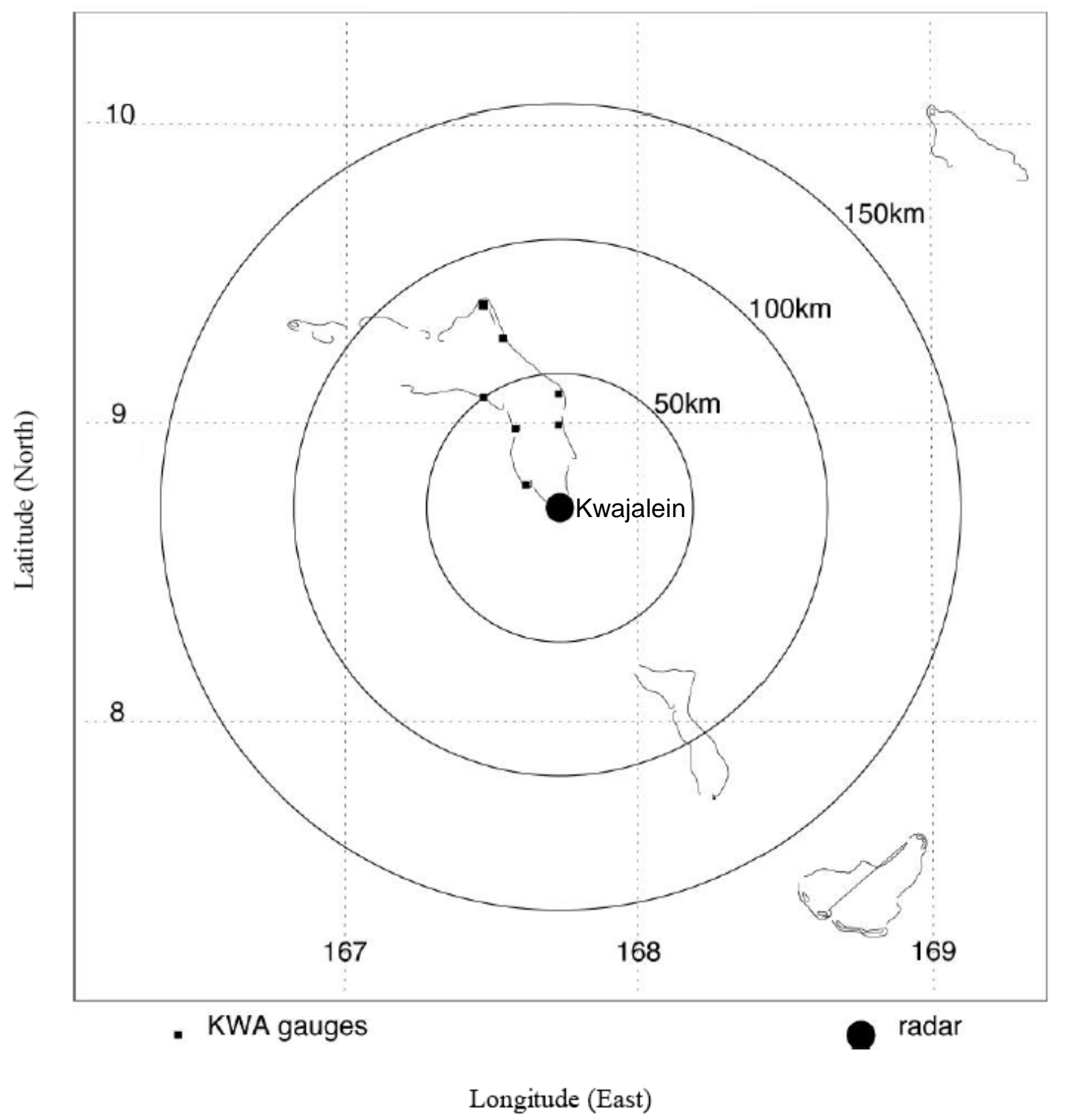

Fig.1: The location of Kwajalein Atoll in the Republic of the Marshall Islands (from Wolff et al. 2005) The KPOL S-band radar is located on Kwajalein Atoll at the center of the image $\left(8.7^{\circ} \mathrm{N}\right.$, $\left.167.7^{\circ} \mathrm{E}\right)$. Rain gauge locations from the KWA network are shown as black squares. 

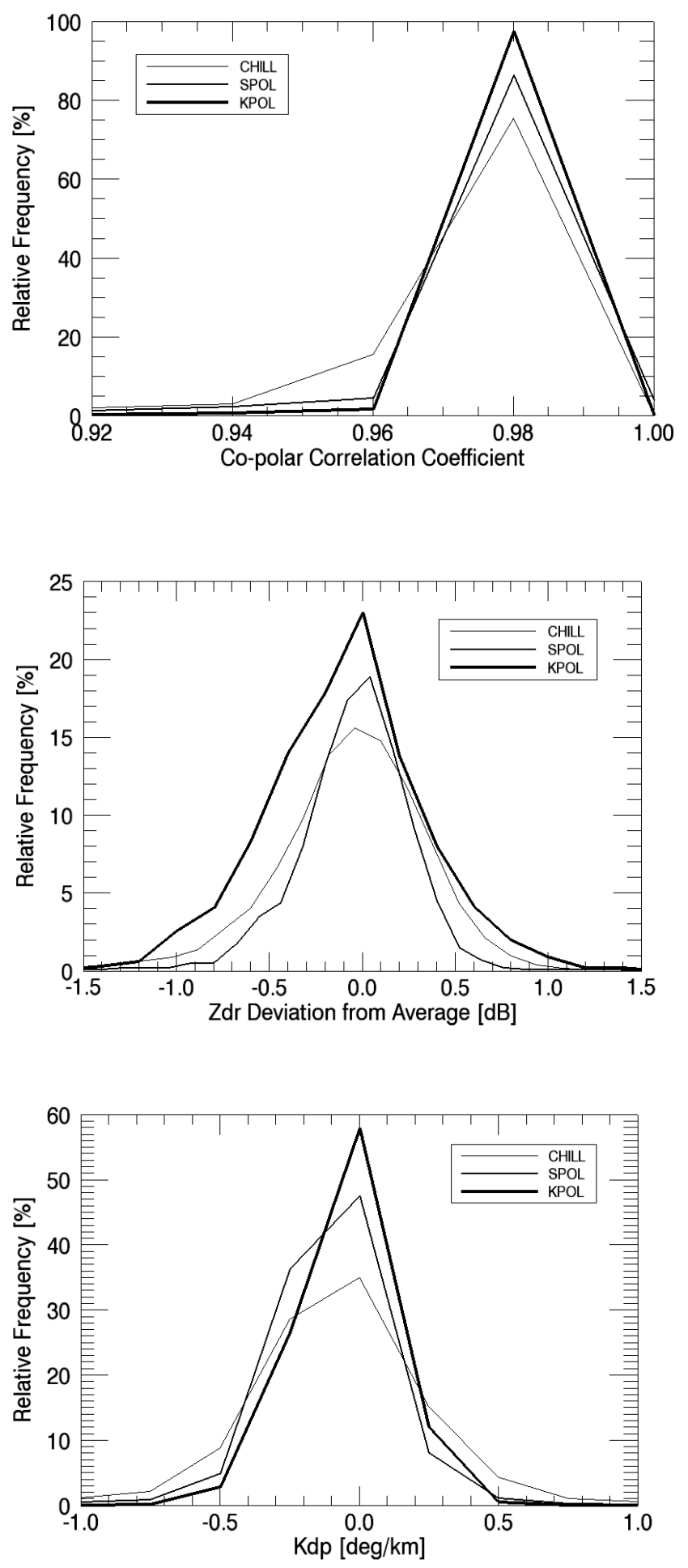

Fig. 2: Comparison of co-polar $\rho_{\mathrm{HV}}$ (top panel), $\mathrm{Z}_{\mathrm{DR}}$ deviation $\left(\mathrm{Z}_{\mathrm{DR}}-\right.$ average $\left.\mathrm{Z}_{\mathrm{DR}}\right)$ (middle), and $\mathrm{K}_{\mathrm{DP}}$ (bottom) polarimetric parameters in light rain from CHILL, SPOL, and KPOL radars. CHILL observations are from 16 July 20042142 UTC, SPOL observations are from 26 Jan. 1999 2139UTC, and KPOL observations are from 11 Aug. 20070352 UTC. 

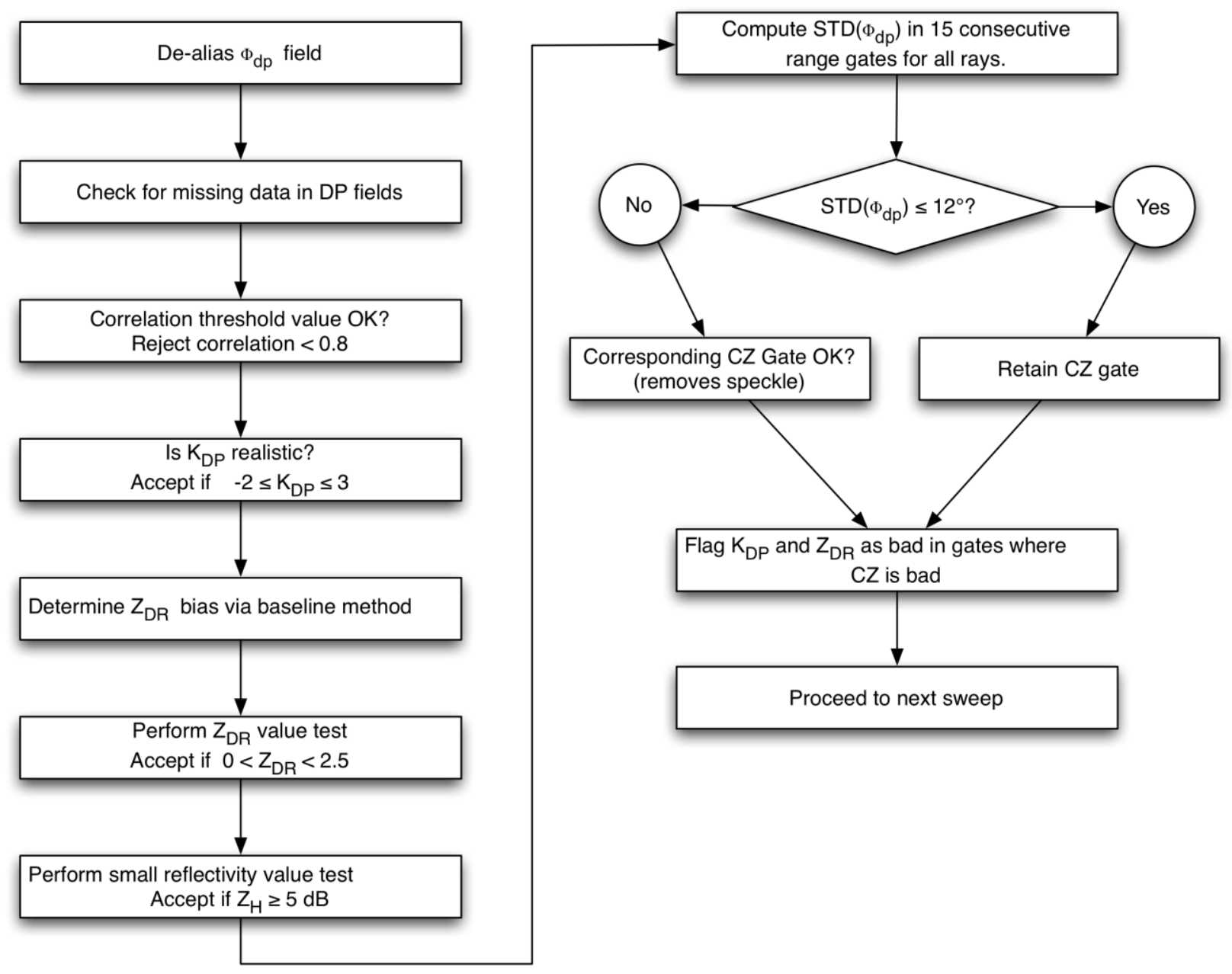

Fig. 3: KPOL quality control algorithm flowchart for the first two elevation angles. All data are below the melting level. 

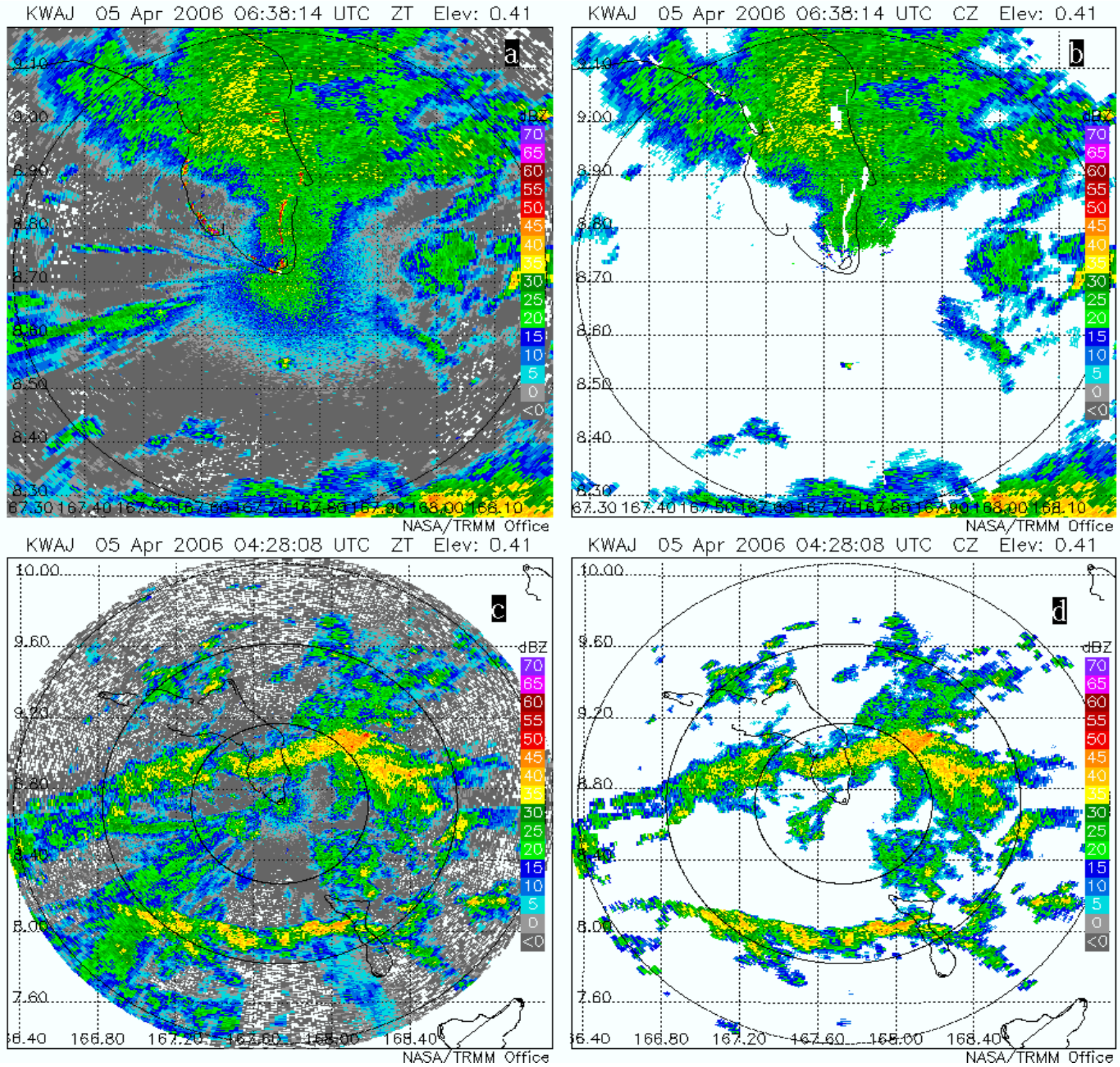

Fig. 4: QC examples showing persistent ground clutter (a) in raw KPOL data along atoll edges (50 km range ring), and removal of embedded and non-embedded ground clutter by the QC algorithm (b). A raw 0.4 degree elevation sweep (c) shows significant multiple-trip echo (southwest of KPOL, 20-160 km range), ground and sea clutter, and typical noise. The QC'd field (d) shows dramatic improvement. Range rings are at $50 \mathrm{~km}$ intervals. 


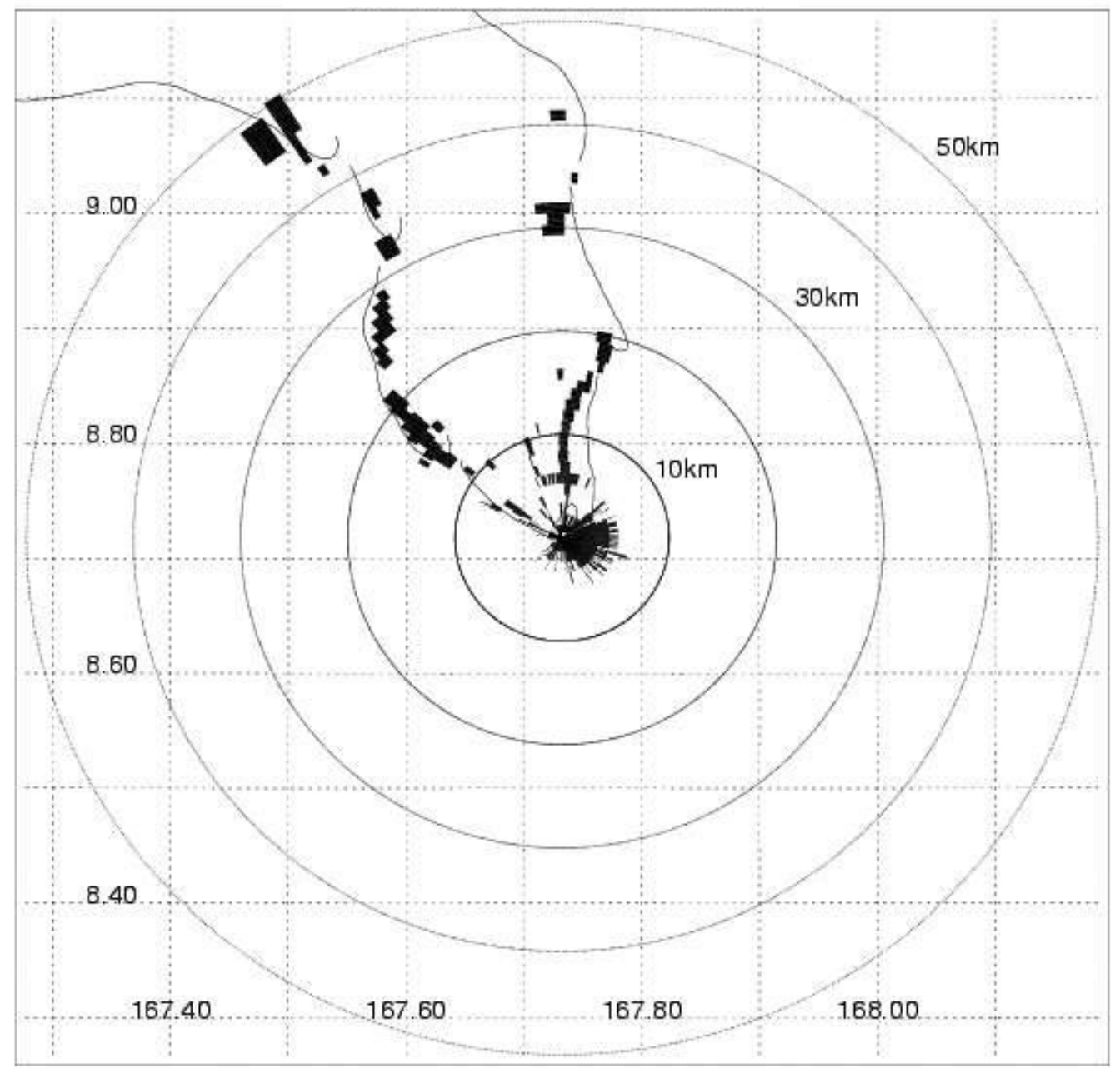

Fig. 5: Map of the clutter field at Kwajalein. Range rings are drawn at 10-km intervals from the radar site. Figure from Silberstein et al. 2008. 

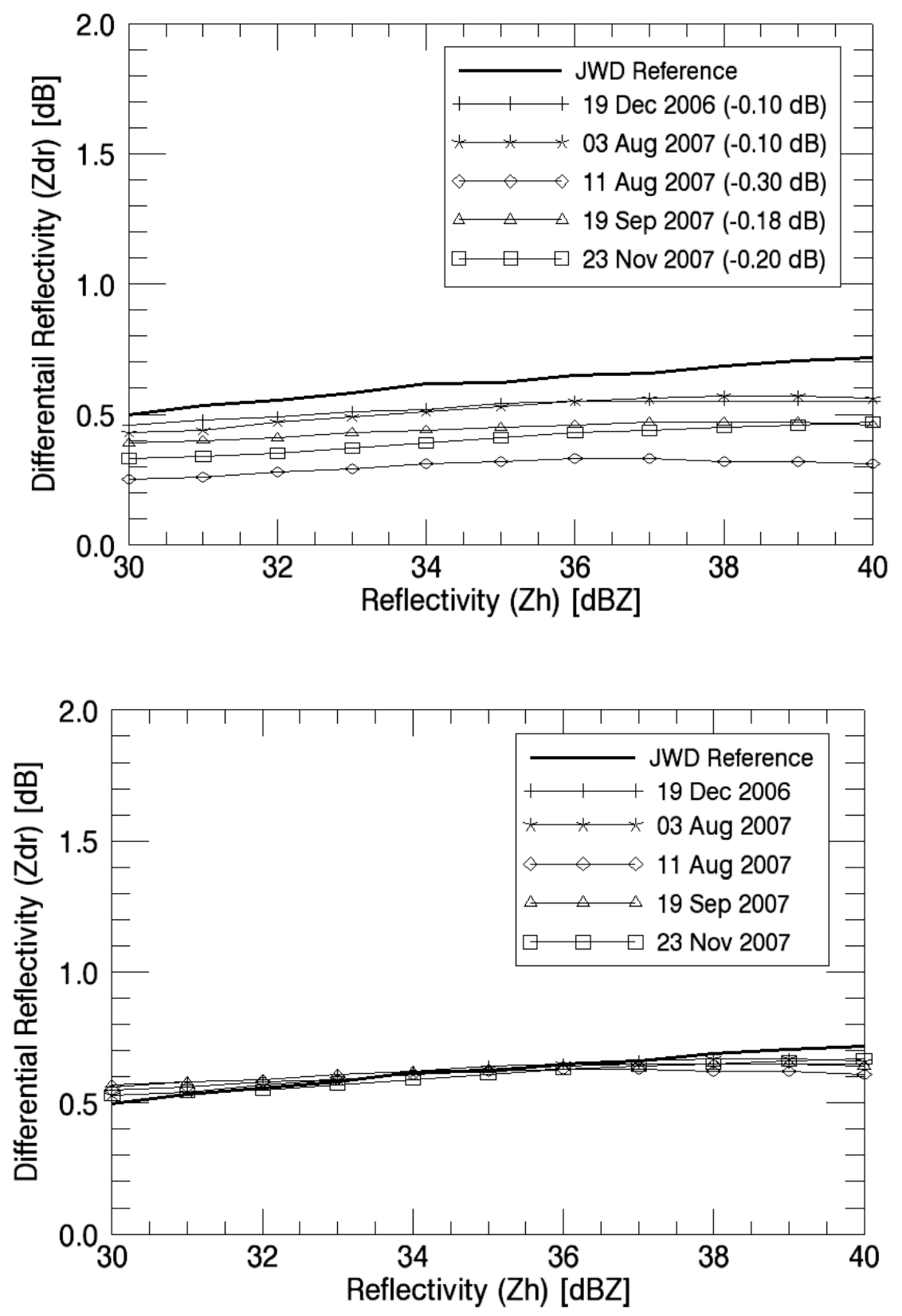

Fig. 6: Distribution of average $Z_{\mathrm{DR}}$ measurements by reflectivity bin. The unadjusted $Z_{\mathrm{DR}}$ curves (top panel) show the bias in five case studies relative to the JWD $Z_{\mathrm{DR}}$ reference (bold, no-symbol line). The bottom panel shows $Z_{\mathrm{DR}}$ distributions bias-adjusted to the JWD reference. $Z_{\mathrm{H}}$ distributions have been calibration corrected via the independent RCA method. 

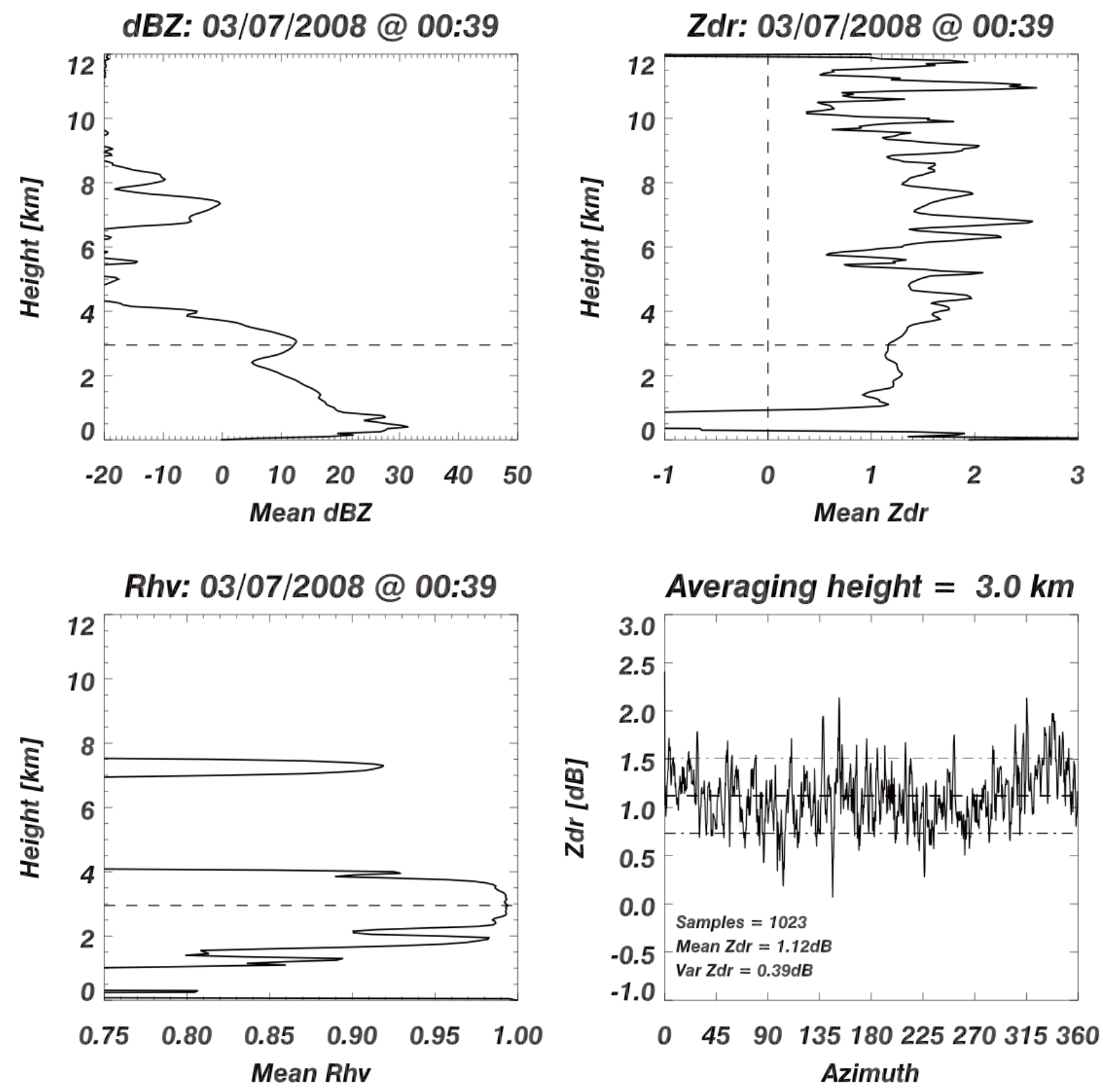

Fig. 7: Measurements of reflectivity with height (top left), mean $Z_{\mathrm{DR}}$ with height (top right) correlation with height (bottom left), and the mean $\mathrm{Z}_{\mathrm{DR}}$ profile with azimuth (bottom right) for a shallow light rain event at Kwajalein from 07 Mar 2008. The correlation profile indicates sampling in the rain medium at the $3.0 \mathrm{~km}$ height. The azimuth profile shows the influence of directional-dependent response. The mean $\mathrm{Z}_{\mathrm{DR}}$ bias of $+1.12 \mathrm{~dB}$ (heavy dashed lined) was determined over the full azimuth cycle. The dash-dot lines represent one standard deviation. 

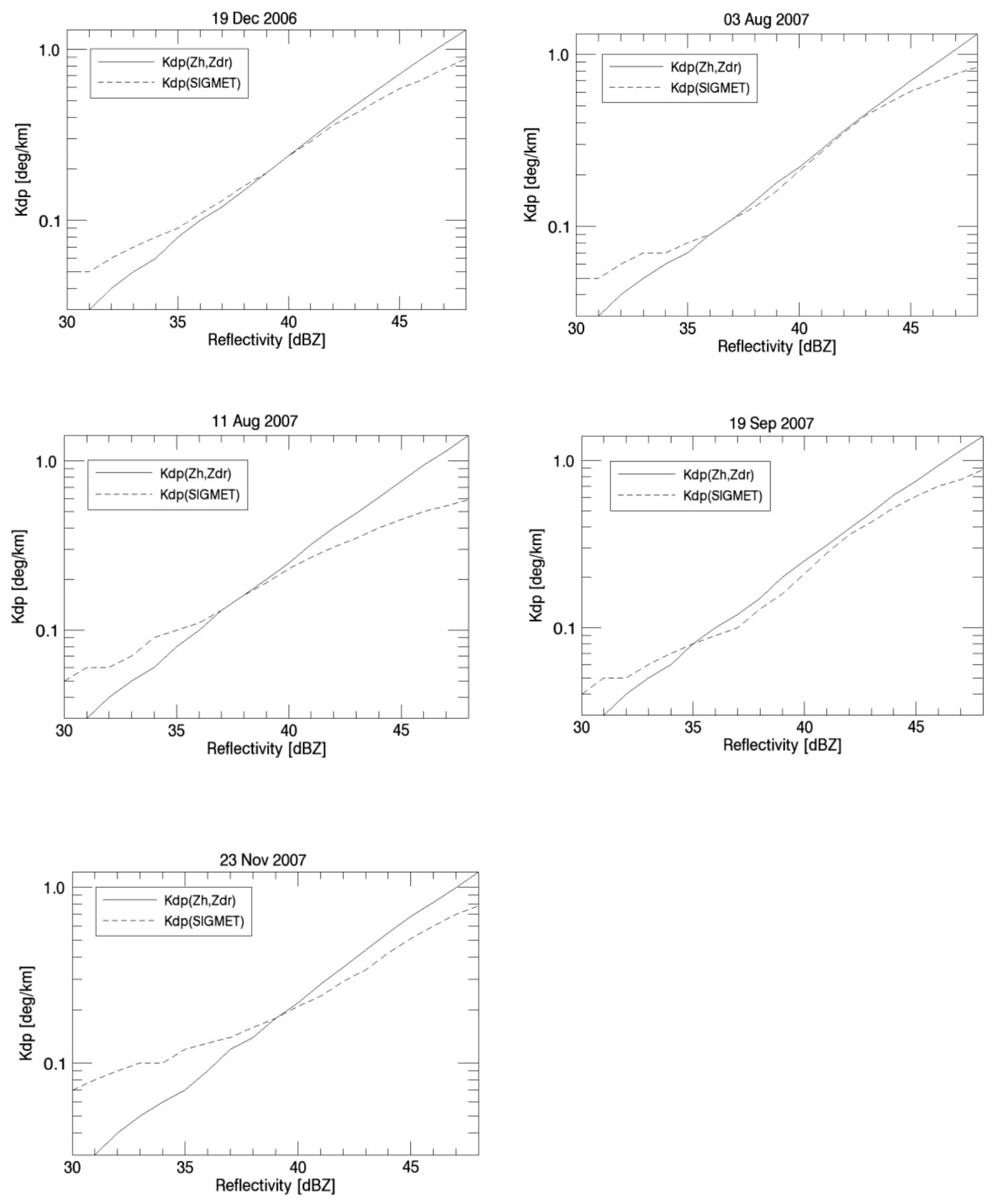

Fig. 8: Comparison of radar processor determined $\mathrm{K}_{\mathrm{DP}}$ measurements and computed $\mathrm{Kdp}$ measurements from self-consistency theory. The self-consistency relationship was developed from disdrometer measurements at Kwajalein. Results are shown from each of the five case studies. 
Kwaj Jul-Dec 2008

Calibration and QC applied

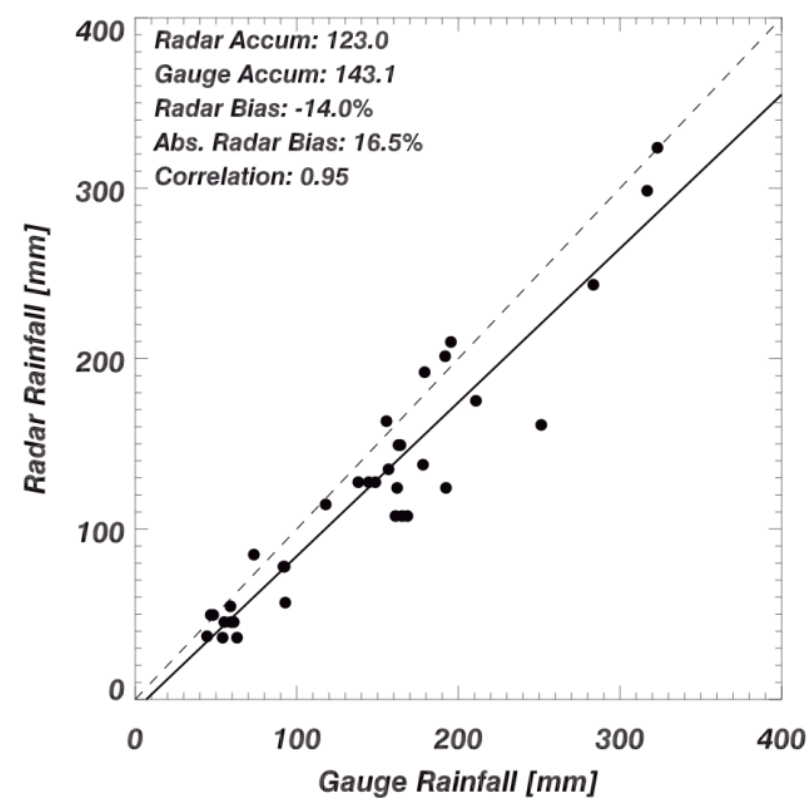

Kwaj Jul-Dec 2008

No calibration; No QC

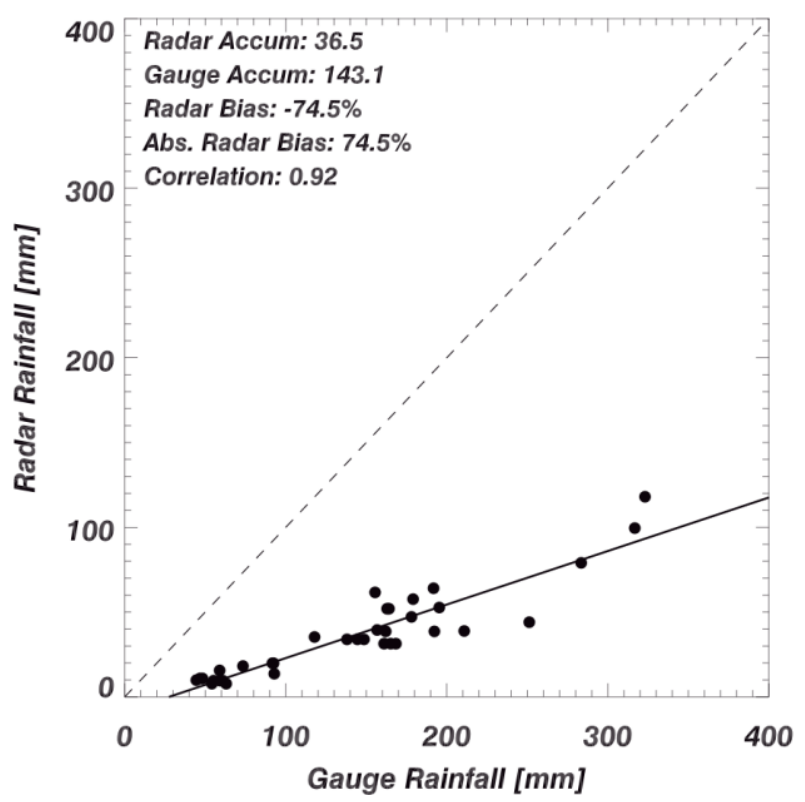

Figure 9: Scatterplots of independent gauge and radar monthly accumulations for the period July-December 2008 at Kwajalein using the polarimetrically-tuned Z-R approach of Bringi et al. (2004). Results show the benefit of quality controlled and calibrated radar data (left panel) vs. uncorrected data (right panel). The absolute bias using corrected and uncorrected radar data are $16.5 \%$ and $74.5 \%$, respectively, with bias calculated as $\frac{\overline{\mid \text { Radar-Gauge }}}{\overline{\text { Gauge }}}$. Regression lines (solid) are shown for each dataset along with the 1-to-1 line. 
Table 1. KPOL Basic Characteristics, Moments Recorded and Field Descriptions

\begin{tabular}{ll}
\hline Processor: & SIGMET RVP8 / RCP8 combination \\
Frequency: & $2.8 \mathrm{GHz}$ \\
Wavelength: & $10.71 \mathrm{~cm}$ \\
Beamwidth: & $1.1^{\circ}$ (horizontal and vertical) \\
& $\begin{array}{l}\text { Linear, horizontal and vertical } \\
\text { Operation Mode: }\end{array}$ \\
& Simultaneous dual transmit and receive (STAR)
\end{tabular}

Moments Recorded:

$\begin{array}{ll}\mathrm{Z}_{\mathrm{H}}: & \text { Reflectivity, horizontal component } \\ \mathrm{V}_{\mathrm{r}}: & \text { Radial velocity } \\ \sigma_{\mathrm{v}}: & \text { Spectrum width } \\ \mathrm{Z}_{\mathrm{DR}}: & \text { Differential reflectivity } \\ \Phi_{\mathrm{DP}}: & \text { Total differential phase } \\ \mathrm{K}_{\mathrm{DP}}: & \text { Specific differential phase (processor computed) } \\ \rho_{\mathrm{HV}}(0): & \text { co-polar cross correlation at zero lag }\end{array}$

Field Variable Descriptions [with associated units] for KPOL data:

ZT [dBZ]: $\quad$ Raw reflectivity, horizontal component

DZ [dBZ]: SIGMET ground-clutter corrected reflectivity, horizontal component

CZ*[dBZ]: Quality controlled reflectivity, horizontal component

VR [ $\left.\mathrm{m} \mathrm{s}^{-1}\right]: \quad$ Radial velocity

DR [dB]: $\quad$ Differential reflectivity

$\mathrm{PH}\left[{ }^{\circ}\right]: \quad \quad$ Total differential phase

$\mathrm{KD}\left[{ }^{\circ} \mathrm{km}^{-1}\right]$ : $\quad$ Specific differential phase

RH [no units]: Co-polar cross correlation

$\mathrm{SW}\left[\mathrm{m} \mathrm{s}^{-1}\right]$ : $\quad$ Spectrum width

SQ [no units]: Signal Quality Index

* The CZ field is added by the NASA TRMM Satellite Validation Office and contains the reflectivity data that have been corrected for non-precipitation echo and absolute calibration error. 
Table 2. Task configuration of the KPOL radar. Columns are task name, radar polarization, elevation angles (deg), and pulse repetition frequency (PRF). Volume scans alternate between A and $\mathrm{B}$, with one surveillance scan between volume scan sets. There are 10 volume scans per hour (5-A scans and 5-B scans). The alternating scanning was replaced with a single 17elevation volume scan with 6 volume scans per hour in October 2008.

\begin{tabular}{|c|c|c|c|}
\hline Task & Polarization & Elevation Angles (deg) & PRF \\
\hline GVVOL_A & Dual & $0.4,1.4,2.3,4.2,6.1,8.0,9.9,11.8,14.0,16.6,19.6,23.2$ & 960 \\
\hline GVVOL_B & Dual & $0.4,1.4,3.3,5.2,7.1,9.0,10.9,12.9,15.2,18.0,21.3,25.3$ & 960 \\
\hline Surv_TRMM & Horizontal & 0.4 & 396 \\
\hline \multicolumn{4}{|c|}{ Volume Scan Strategy beginning October 2008 (Alternating volumes A and B replaced) } \\
\hline GVVOL & Dual & $\begin{array}{l}0.4,1.5,2.6,3.7,4.8,5.9,7.0,8.1,9.2,10.3,11.6,13.2 \\
15.2,17.7,20.8,24.6,29.2,34.7\end{array}$ & 960 \\
\hline
\end{tabular}


Table 3 Empirical investigation of relative DP radar performance in light rain. For both CHILL and SPOL radars, one case was selected for comparison. For KPOL, 12 cases from 2006 and 2007 were analyzed. The KPOL statistics represent the range of values observed from these cases. The standard deviation of the measured differential phase $\sigma\left(\Phi_{\mathrm{DP}}\right)$ was computed at each range gate from a running, centered 21 gate sample for CHILL and SPOL, and a 25 gate sample for KPOL corresponding to the length scale used for $\mathrm{K}_{\mathrm{DP}}$ calculation. The average absolute deviation of $\mathrm{Z}_{\mathrm{DR}}\left(\mathrm{AAD} \mathrm{Z}_{\mathrm{DR}}\right.$ - see equation 2) represents an upper-end estimate of the random measurement error.

\begin{tabular}{|c|c|c|c|c|c|c|c|}
\hline $\begin{array}{l}\text { Radar } \\
\text { Name }\end{array}$ & $\begin{array}{c}\text { Date/Time }(Z) \\
\text { YYMMDDHHMM }\end{array}$ & \# of Gates & $\begin{array}{c}\text { Median } Z_{\mathrm{H}} \\
\mathrm{dBZ} \\
\end{array}$ & $\begin{array}{c}\text { Median } \mathrm{K}_{\mathrm{DP}} \\
{\text { deg } \mathrm{km}^{-1}}\end{array}$ & Median $\rho_{\mathrm{HV}}$ & $\begin{array}{c}\text { Median } \sigma(\Phi D P) \\
\text { deg }\end{array}$ & $\begin{array}{c}\mathrm{AAD} \mathrm{Z}_{\mathrm{L}} \\
\mathrm{dB}\end{array}$ \\
\hline CHILL & 0407162142 & 4946 & 22.6 & 0.0 & 0.99 & 2.5 & 0.4 \\
\hline SPOL & 9901262139 & 6827 & 22.5 & 0.0 & 0.99 & 1.4 & 0.3 \\
\hline KPOL & 12 cases & $3900-25000$ & $20-28$ & 0.0 & $\geq 0.98$ & $2.2-3.1$ & $0.3-0.4$ \\
\hline
\end{tabular}


Table 4. Case studies and time periods selected (HHMM UTC) for application of the DP QC algorithm. Statistics represent the maximum percentage of gates flagged as "bad" or "noise" by the individual QC tests from the first two elevation angles within each volume scan. Statistics are from the rainfall estimation range of $0-100 \mathrm{~km}$. Gates flagged in total reflectivity (ZT) includes those from the $\rho_{\mathrm{HV}}, \sigma\left(\Phi_{\mathrm{DP}}\right)$, low reflectivity threshold, and missing gate tests from the DP fields. The ZT percentages vary significantly from case-to-case and are heavily influenced by the consideration of gates less than $5 \mathrm{dBZ}$ as "noise".

\begin{tabular}{lccccc} 
Case & $\rho \mathrm{HV}$ & KDP & ZDR & $\sigma(\Phi \mathrm{DP})$ & Total ZT \\
\hline $\begin{array}{l}\text { 19 Dec 2006 } \\
\text { 0544-0637 UTC }\end{array}$ & 3 & 1 & 17 & 2 & 5 \\
03 Aug 2007 & 2 & 2 & 34 & 3 & 8 \\
0200-0453 UTC & & & & & \\
11 Aug 2007 & 8 & 3 & 30 & 6 & 29 \\
0325-0439 UTC & & & & & \\
19 Sep 2007 & 4 & 2 & 33 & 4 & 24 \\
1542-1729 UTC & & & & & \\
23 Nov 2007 & 5 & 2 & 23 & 7 & 29 \\
1603-1730 UTC & & & & &
\end{tabular}


Table 5. Comparison of DP and non-polarimetric TRMM GVS QC results with respect to ground clutter detection. Raw reflectivity data were extracted from locations with frequent ground clutter echoes. The gate count represents the remaining gates considered to be ground clutter after QC is applied. For this study, gates $\geq 55 \mathrm{dBZ}$ are considered ground clutter. DP QC shows superior performance as compared to the non-polarimetric (TRMM GVS) QC as evidenced by the low gate counts.

\begin{tabular}{|c|c|c|c|c|c|c|}
\hline Case & $\begin{array}{l}\text { Number of unedited gates } \\
\geq 55 \mathrm{dBZ} / \text { total extracted gates }\end{array}$ & $\begin{array}{r}\text { DP } \\
\text { Gate } \mathrm{C} \\
\mathrm{dBZ} \geq 0\end{array}$ & & $\begin{array}{r}\mathrm{G} \\
\mathrm{Gat} \\
\geq 55 \mathrm{~dB}\end{array}$ & $\begin{array}{l}\text { QC } \\
\text { Coun } \\
\geq 60\end{array}$ & 65 \\
\hline 19 Dec 2006 & $71 / 11907$ & 0 & 0 & 53 & 3 & 0 \\
\hline 03 Aug 2007 & 237 / 35721 & 3 & 0 & 193 & 45 & 0 \\
\hline 11 Aug 2007 & 383 / 15720 & 0 & 0 & 85 & 31 & 0 \\
\hline 19 Sep 2007 & 137 / 18522 & 0 & 0 & 111 & 28 & 0 \\
\hline 23 Nov 2007 & 528 / 18429 & 9 & 0 & 478 & 166 & 34 \\
\hline
\end{tabular}


Table 6. Reflectivity bin statistics to determine $\mathrm{K}_{\mathrm{DP}}$ data validity from the 19 Dec 2006 case study. Shown are the reflectivity bin, average $\mathrm{K}_{\mathrm{DP}}$ within the bin, number of independent $\mathrm{K}_{\mathrm{DP}}$ samples, times reduction in $\mathrm{K}_{\mathrm{DP}}$ standard error due to averaging, maximum allowed standard deviation of $\mathrm{K}_{\mathrm{DP}}$, and the standard deviation range of $\mathrm{K}_{\mathrm{DP}}$ within the bin.

\begin{tabular}{|c|c|c|c|c|c|}
\hline $\mathrm{dBZ}$ Bin & $\begin{array}{l}\text { Kdp Avg } \\
\left({ }^{\circ} \mathrm{km}^{-1}\right)\end{array}$ & $\begin{array}{l}\text { \#indep Kdp } \\
\text { Samples }\end{array}$ & $\begin{array}{l}\text { Times } \\
\text { Reduction }\end{array}$ & $\begin{array}{l}\text { Max allowed } \\
\sigma(\mathrm{Kdp})\left({ }^{\circ} \mathrm{km}^{-1}\right)\end{array}$ & $\begin{array}{c}\text { Observed Data Range } \\
\sigma(\mathrm{Kdp})\left({ }^{\circ} \mathrm{km}^{-1}\right)\end{array}$ \\
\hline 30.0 & 0.053 & 1741 & 41 & 0.011 & $0.004-0.006$ \\
\hline 31.0 & 0.059 & 1833 & 42 & 0.012 & $0.004-0.006$ \\
\hline 32.0 & 0.066 & 1915 & 43 & 0.013 & $0.004-0.005$ \\
\hline 33.0 & 0.076 & 1957 & 44 & 0.015 & $0.004-0.005$ \\
\hline 34.0 & 0.088 & 1906 & 43 & 0.018 & $0.004-0.005$ \\
\hline 35.0 & 0.102 & 1790 & 42 & 0.020 & $0.004-0.006$ \\
\hline 36.0 & 0.120 & 1615 & 40 & 0.024 & $0.004-0.006$ \\
\hline 37.0 & 0.141 & 1402 & 37 & 0.028 & $0.005-0.006$ \\
\hline 38.0 & 0.170 & 1158 & 34 & 0.034 & $0.005-0.007$ \\
\hline 39.0 & 0.210 & 945 & 30 & 0.042 & $0.006-0.008$ \\
\hline 40.0 & 0.260 & 763 & 27 & 0.052 & $0.006-0.009$ \\
\hline 41.0 & 0.321 & 616 & 24 & 0.064 & $0.007-0.010$ \\
\hline 42.0 & 0.391 & 501 & 22 & 0.078 & $0.008-0.011$ \\
\hline 43.0 & 0.457 & 402 & 20 & 0.091 & $0.008-0.012$ \\
\hline 44.0 & 0.543 & 309 & 17 & 0.109 & $0.010-0.014$ \\
\hline 45.0 & 0.626 & 236 & 15 & 0.125 & $0.011-0.016$ \\
\hline 46.0 & 0.708 & 176 & 14 & 0.142 & $0.013-0.018$ \\
\hline 47.0 & 0.822 & 127 & 12 & 0.164 & $0.015-0.021$ \\
\hline 48.0 & 0.913 & 79 & 10 & 0.183 & $0.019-0.027$ \\
\hline
\end{tabular}


Table 7. Self-consistency derived calibration adjustments as compared with the independent RCA approach, and the absolute value of their difference. Shown is the cumulative number of $\mathrm{K}_{\mathrm{DP}}$ samples compared (measured and derived via consistency) from the reflectivity bins in the $\mathrm{Zmin}(30 \mathrm{dBZ})$ to $\mathrm{Zmax}(48 \mathrm{dBZ})$ range.

\begin{tabular}{lcccc} 
Case & $\begin{array}{c}\text { Kdp } \\
\text { samples }\end{array}$ & $\begin{array}{c}\text { Self-Consistency Calibration } \\
Z_{\text {BIAS }}(\mathrm{dB})\end{array}$ & $\begin{array}{l}\text { RCA } \\
(\mathrm{dB})\end{array}$ & $\begin{array}{c}\text { Difference } \\
(\mathrm{dB})\end{array}$ \\
\hline 19 Dec 2006 & 546434 & -2.44 & -1.95 & 0.49 \\
03 Aug 2007 & 1521223 & -2.06 & -1.78 & 0.28 \\
11 Aug 2007 & 335658 & -1.46 & -1.91 & 0.45 \\
19 Sep 2007 & 400012 & -0.93 & -1.91 & 0.98 \\
23 Nov 2007 & 740603 & -2.17 & -2.46 & 0.29
\end{tabular}

